\title{
Representation of the Solutions of Linear Discrete Systems with Constant Coefficients and Two Delays
}

\author{
Josef Diblík $^{1,2}$ and Blanka Morávkováa ${ }^{1,2}$ \\ ${ }^{1}$ Department of Mathematics and Descriptive Geometry, Faculty of Civil Engineering, Brno University of Technology, \\ 60200 Brno, Czech Republic \\ ${ }^{2}$ Department of Mathematics, Faculty of Electrical Engineering, Brno University of Technology, 61600 Brno, Czech Republic
}

Correspondence should be addressed to Josef Diblík; diblik@feec.vutbr.cz

Received 5 September 2013; Accepted 4 October 2013; Published 16 April 2014

Academic Editor: Miroslava Růžičková

Copyright (C) 2014 J. Diblík and B. Morávková. This is an open access article distributed under the Creative Commons Attribution License, which permits unrestricted use, distribution, and reproduction in any medium, provided the original work is properly cited.

The purpose of this paper is to develop a method for the construction of solutions to initial problems of linear discrete systems with constant coefficients and with two delays $\Delta x(k)=B x(k-m)+C x(k-n)+f(k)$, where $m, n \in \mathbb{N}, m \neq n$, are fixed, $k=0, \ldots, \infty$, $B=\left(b_{i j}\right), C=\left(c_{i j}\right)$ are constant $r \times r$ matrices, $f$ is a given $r \times 1$ vector, and $x$ is an $r \times 1$ unknown vector. Solutions are expressed with the aid of a special function called the discrete matrix delayed exponential for two delays. Such approach results in a possibility to express an initial Cauchy problem in a closed form. Examples are shown illustrating the results obtained.

\section{Introduction}

Throughout the paper, we will use the following notation. For integers $s, t, s \leq t$, we define the set $\mathbb{Z}_{s}^{t}:=\{s, s+1, \ldots, t-1, t\}$. Similarly, we define the sets $\mathbb{Z}_{-\infty}^{t}:=\{\ldots, t-1, t\}$ and $\mathbb{Z}_{s}^{\infty}:=$ $\{s, s+1, \ldots\}$. The function $\lfloor\cdot\rfloor$ used below is the floor integer function. We will employ the following property of the floor integer function:

$$
x-1<\lfloor x\rfloor \leq x,
$$

where $x \in \mathbb{R}$.

In this paper, we deal with the discrete system

$$
\Delta x(k)=B x(k-m)+C x(k-n)+f(k),
$$

where $m, n \in \mathbb{N}, m \neq n$, are fixed, $k \in \mathbb{Z}_{0}^{\infty}, B=\left(b_{i j}\right), C=\left(c_{i j}\right)$ are constant $r \times r$ matrices, $f: \mathbb{Z}_{0}^{\infty} \rightarrow \mathbb{R}^{r}$ is a given $r \times 1$ vector, and $x: \mathbb{Z}_{0}^{\infty} \rightarrow \mathbb{R}^{r}$ is an $r \times 1$ unknown vector.
Together with (2), we consider an initial (Cauchy) problem

$$
x(k)=\varphi(k) .
$$

Define binomial coefficients as customary; that is, for $n \in$ $\mathbb{Z}$ and $k \in \mathbb{Z}$,

$$
\left(\begin{array}{l}
n \\
k
\end{array}\right):= \begin{cases}\frac{n !}{k !(n-k) !} & \text { if } n \geq k \geq 0 \\
0 & \text { otherwise. }\end{cases}
$$

We recall that, for a well-defined discrete function $\omega(k)$, the forward difference operator $\Delta$ is defined as $\Delta \omega(k)=\omega(k+$ 1) $-\omega(k)$. In the paper, we also adopt the customary notation $\sum_{i=i_{1}}^{i_{2}} g_{i}=0$ if $i_{2}<i_{1}$. In the case of double sums, we set

$$
\sum_{i=i_{1}, j=j_{1}}^{i_{2}, j_{2}} g_{i j}=0
$$

if at least one of the inequalities $i_{2}<i_{1}, j_{2}<j_{1}$ holds. 
In $[1,2]$, a discrete matrix delayed exponential for a single delay $m \in \mathbb{N}$ was defined.

Definition 1. For an $r \times r$ constant matrix $B, k \in \mathbb{Z}$, and fixed $m \in \mathbb{N}$, one defines the discrete matrix delayed exponential $\mathrm{e}_{m}^{B k}$ as follows:

$$
\mathrm{e}_{m}^{B k}:=\left\{\begin{array}{l}
\Theta \\
\quad \text { if } k \in \mathbb{Z}_{-\infty}^{-m-1}, \\
I+\sum_{j=1}^{\ell} B^{j} \cdot\left(\begin{array}{c}
k-m(j-1) \\
j
\end{array}\right) \\
\quad \text { if } \ell=0,1,2, \ldots, k \in \mathbb{Z}_{(\ell-1)(m+1)+1}^{\ell(m+1)},
\end{array}\right.
$$

where $\Theta$ is an $r \times r$ null matrix and $I$ is an $r \times r$ unit matrix.

Such discrete matrix delayed exponential was used in [1] to construct solutions of the initial problems (2), (3) with $C \equiv \Theta$, where $\Theta$ is an $r \times r$ zero matrix. In these constructions, the main property (Theorem 2) of discrete matrix delayed exponential for a single delay $m \in \mathbb{N}$ was utilized in [1].

Theorem 2. Let $B$ be a constant $r \times r$ matrix. Then, for $k \in$ $\mathbb{Z}_{-m}^{\infty}$,

$$
\Delta e_{m}^{B k}=B e_{m}^{B(k-m)} .
$$

The properties of delayed matrix exponential functions for their continuous and discrete variants and their applications are the topic of recent papers [1-18]. We note that the definition of the delayed matrix exponential was first defined for the continuous case in [4] and, for the discrete case, in $[1,2]$.

The paper is organized as follows. Discrete matrix delayed exponentials for two delays and their main property are considered in Section 2. A representation of the solution to problem (2), (3) is given in Section 3 and examples illustrating the results obtained are shown in Section 4.

\section{Discrete Matrix Delayed Exponential for Two Delays and Its Main Property}

In order to extend the results proved in $[1,2]$ to problems (2), (3), a discrete matrix delayed exponential for two delays was proposed in [3]. There is a discrete matrix delayed exponential for two delays $m, n \in \mathbb{N}, m \neq n$, defined as follows.

Definition 3. Let $B, C$ be constant $r \times r$ matrices with $B C=C B$ and let $m, n \in \mathbb{N}, m \neq n$, be fixed integers. One defines a discrete $r \times r$ matrix function $\mathrm{e}_{m n}^{B C k}$ called the discrete matrix delayed exponential for two delays $m, n$ and for two $r \times r$ constant matrices $B, C$ :

$$
\begin{aligned}
& \mathrm{e}_{m n}^{B C k} \\
& := \begin{cases}\Theta & \text { if } k \in \mathbb{Z}_{-\infty}^{-\max \{m, n\}-1}, \\
I & \text { if } k \in \mathbb{Z}_{-\max \{m, n\}}^{0}, \\
I+(B+C) \sum_{i=0, j=0}^{p_{(k)}^{-1, q_{(k)}-1}} B^{i} C^{j}\left(\begin{array}{c}
i+j \\
i
\end{array}\right)\left(\begin{array}{c}
k-m i-n j \\
i+j+1
\end{array}\right) & \\
& \text { if } k \in \mathbb{Z}_{1}^{\infty},\end{cases}
\end{aligned}
$$

where

$$
p_{(k)}:=\left\lfloor\frac{k+m}{m+1}\right\rfloor, \quad q_{(k)}:=\left\lfloor\frac{k+n}{n+1}\right\rfloor .
$$

Let us show an example illustrating this special exponential function.

Example 4. For $k \in \mathbb{Z}_{0}^{12}$ we will construct the matrix $\mathrm{e}_{m n}^{B C k}$ if $m=2$ and $n=3$. Computing particular matrices generating $\mathrm{e}_{2,3}^{B C k}$ for $k \in \mathbb{Z}_{0}^{12}$, we get

$$
\begin{aligned}
& \mathrm{e}_{2,3}^{B C 0}=I, \quad \mathrm{e}_{2,3}^{B C 1}=I+B+C, \\
& \mathrm{e}_{2,3}^{B C 2}=I+(B+C) 2, \quad \mathrm{e}_{2,3}^{B C 3}=I+(B+C) 3 \\
& \mathrm{e}_{2,3}^{B C 4}=I+(B+C)(4+B) \\
& \mathrm{e}_{2,3}^{B C 5}=I+(B+C)(5+3 B+C) \\
& \mathrm{e}_{2,3}^{B C 6}=I+(B+C)(6+6 B+3 C) \\
& \mathrm{e}_{2,3}^{B C 7}=I+(B+C)\left(7+10 B+6 C+B^{2}\right) \\
& e_{2,3}^{B C 8}=I+(B+C)\left(8+15 B+10 C+4 B^{2}+2 B C\right) \\
& e_{2,3}^{B C 9}=I+(B+C)\left(9+21 B+15 C+10 B^{2}+8 B C+C^{2}\right) \\
& e_{2,3}^{B C 10}=I+(B+C) \\
& \quad \times\left(10+28 B+21 C+20 B^{2}+20 B C+4 C^{2}+B^{3}\right) \\
& e_{2,3}^{B C 12}=I+(B+C)\left(12+45 B+36 C+56 B^{2}+70 B C\right. \\
& e_{2,3}^{B C 11}=I+(B+C)\left(11+36 B+28 C+35 B^{2}\right. \\
& \left.\quad+40 B C+10 C^{2}+5 B^{3}+3 B^{2} C\right) \\
& \left.\quad+15 B^{3}+15 B^{2} C+3 B C^{2}\right)
\end{aligned}
$$

The main property of $\mathrm{e}_{m n}^{B C k}$ was proved in [3]. 
Theorem 5. Let $B, C$ be constant $r \times r$ matrices with $B C=C B$ and let $m, n \in \mathbb{N}, m \neq n$, be fixed integers. Then

$$
\Delta e_{m n}^{B C k}=B e_{m n}^{B C(k-m)}+C e_{m n}^{B C(k-n)}
$$

for $k \in \mathbb{Z}_{0}^{\infty}$.

The analysis of $e_{m n}^{B C k}$ applicability to a representation of the solution to initial problem (2), (3) unfortunately does not lead to satisfactory results because, as we will see below, an additional condition $\operatorname{det}(B+C) \neq 0$ is necessary. A small difference in the definition results in representations of solutions of initial problems without this assumption. Now we give a second definition of a discrete matrix delayed exponential for two delays $\widetilde{\mathrm{e}}_{m n}^{B C k}$.

Definition 6. Let $B, C$ be constant $r \times r$ matrices with $B C=C B$ and let $m, n \in \mathbb{N}, m<n$, be fixed integers. One defines a discrete $r \times r$ matrix function $\widetilde{\mathrm{e}}_{m n}^{B C k}$ called the discrete matrix delayed exponential for two delays $m, n$ and for two $r \times r$ constant matrices $B, C$ as follows:

$$
\begin{aligned}
& \widetilde{\mathrm{e}}_{m n}^{B C k}
\end{aligned}
$$

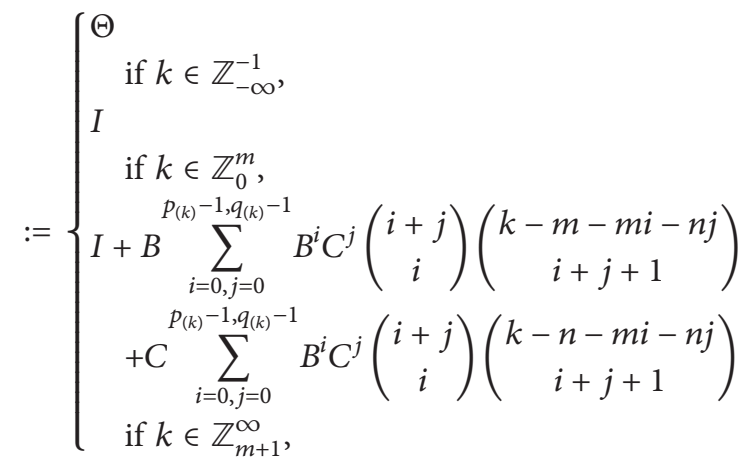

where

$$
p_{(k)}:=\left\lfloor\frac{k+m}{m+1}\right\rfloor, \quad q_{(k)}:=\left\lfloor\frac{k+n}{n+1}\right\rfloor .
$$

Remark 7. For $k \in \mathbb{Z}_{0}^{n}$, it is easy to deduce that $\widetilde{\mathrm{e}}_{m n}^{B C k}=\mathrm{e}_{m}^{B(k-m)}$.

In order to compare both types of discrete delayed matrices for two delays and see the difference between both definitions, we consider the following example where delays are the same as in Example 4.

Example 8. For $k \in \mathbb{Z}_{0}^{12}$ we will construct the matrix $\widetilde{\mathrm{e}}_{m n}^{B C k}$ if $m=2$ and $n=3$. Computing particular matrices generating $\widetilde{\mathrm{e}}_{2,3}^{B C k}$ for $k \in \mathbb{Z}_{0}^{12}$, we get

$$
\begin{aligned}
& \widetilde{\mathrm{e}}_{2,3}^{B C 0}=I, \quad \widetilde{\mathrm{e}}_{2,3}^{B C 1}=I, \\
& \widetilde{\mathrm{e}}_{2,3}^{B C 2}=I, \quad \widetilde{\mathrm{e}}_{2,3}^{B C 3}=I+B, \\
& \widetilde{\mathrm{e}}_{2,3}^{B C 4}=I+2 B+C, \quad \widetilde{\mathrm{e}}_{2,3}^{B C 5}=I+3 B+2 C,
\end{aligned}
$$

$$
\begin{aligned}
\widetilde{\mathrm{e}}_{2,3}^{B C 6}=I+4 B+3 C+B^{2} \\
\widetilde{\mathrm{e}}_{2,3}^{B C 7}=I+5 B+4 C+3 B^{2}+2 B C \\
\widetilde{\mathrm{e}}_{2,3}^{B C 8}=I+6 B+5 C+6 B^{2}+6 B C+C^{2} \\
\widetilde{\mathrm{e}}_{2,3}^{B C 9}=I+7 B+6 C+10 B^{2}+12 B C+3 C^{2}+B^{3}, \\
\widetilde{\mathrm{e}}_{2,3}^{B C 10}=I+8 B+7 C+15 B^{2}+20 B C+6 C^{2}+4 B^{3}+3 B^{2} C, \\
\widetilde{\mathrm{e}}_{2,3}^{B C 11}=I+9 B+8 C+21 B^{2}+30 B C+10 C^{2} \\
\quad+10 B^{3}+12 B^{2} C+3 B C^{2}, \\
\widetilde{\mathrm{e}}_{2,3}^{B C 12=}=I+10 B+9 C+28 B^{2}+42 B C+15 C^{2} \\
\quad+20 B^{3}+30 B^{2} C+12 B C^{2}+C^{3}+B^{4} .
\end{aligned}
$$

The main property of $\widetilde{\mathrm{e}}_{m n}^{B C k}$ is given by the following theorem.

Theorem 9. Let $B, C$ be constant $r \times r$ matrices with $B C=C B$ and let $m, n \in \mathbb{N}, m<n$, be fixed integers. Then

$$
\Delta \widetilde{\mathrm{e}}_{m n}^{B C k}=B \widetilde{\mathrm{e}}_{m n}^{B C(k-m)}+C \widetilde{\mathrm{e}}_{m n}^{B C(k-n)}
$$

for $k \in \mathbb{Z}_{0}^{\infty}$.

Proof. Let $k \geq 1$. From (1) and (13), we can see easily that, for an integer $k \geq 0$ satisfying

$$
\begin{aligned}
\left(p_{(k)}\right. & -1)(m+1)+1 \\
& \leq k \leq p_{(k)}(m+1) \wedge\left(q_{(k)}-1\right)(n+1)+1 \\
& \leq k \leq q_{(k)}(n+1),
\end{aligned}
$$

the equation

$$
\begin{aligned}
& \Delta \widetilde{\mathrm{e}}_{m n}^{B C k}=\Delta\left[I+B \sum_{i=0, j=0}^{p_{(k)}-1, q_{(k)}-1} B^{i} C^{j}\left(\begin{array}{c}
i+j \\
i
\end{array}\right)\left(\begin{array}{c}
k-m-m i-n j \\
i+j+1
\end{array}\right)\right. \\
& \left.+C \sum_{i=0, j=0}^{p_{(k)}-1, q_{(k)}-1} B^{i} C^{j}\left(\begin{array}{c}
i+j \\
i
\end{array}\right)\left(\begin{array}{c}
k-n-m i-n j \\
i+j+1
\end{array}\right)\right]
\end{aligned}
$$

holds by Definition 6 of $\widetilde{\mathrm{e}}_{m n}^{B C k}$. Since $\Delta I=\Theta$, we have

$$
\begin{aligned}
\Delta \widetilde{\mathrm{e}}_{m n}^{B C k}=\Delta\left[B \sum_{i=0, j=0}^{p_{(k)}-1, q_{(k)}-1} B^{i} C^{j}\left(\begin{array}{c}
i+j \\
i
\end{array}\right)\left(\begin{array}{c}
k-m-m i-n j \\
i+j+1
\end{array}\right)\right. \\
\left.+C \sum_{i=0, j=0}^{p_{(k)}-1, q_{(k)}-1} B^{i} C^{j}\left(\begin{array}{c}
i+j \\
i
\end{array}\right)\left(\begin{array}{c}
k-n-m i-n j \\
i+j+1
\end{array}\right)\right] .
\end{aligned}
$$

By the definition of the forward difference, that is,

$$
\Delta \widetilde{\mathrm{e}}_{m n}^{B C k}=\widetilde{\mathrm{e}}_{m n}^{B C(k+1)}-\widetilde{\mathrm{e}}_{m n}^{B C k},
$$


we conclude that it is reasonable to divide the proof into four parts given by the four values of integer $k$.

In the first case, $k$ is such that

$$
\begin{aligned}
\left(p_{(k)}\right. & -1)(m+1)+1 \\
& \leq k<p_{(k)}(m+1) \wedge\left(q_{(k)}-1\right)(n+1)+1 \\
& \leq k<q_{(k)}(n+1),
\end{aligned}
$$

in the second case

$$
k=p_{(k)}(m+1) \wedge\left(q_{(k)}-1\right)(n+1)+1 \leq k<q_{(k)}(n+1),
$$

in the third case

$$
\left(p_{(k)}-1\right)(m+1)+1 \leq k<p_{(k)}(m+1) \wedge k=q_{(k)}(n+1),
$$

and in the fourth case

$$
k=p_{(k)}(m+1) \wedge k=q_{(k)}(n+1) .
$$

We see that the above four cases cover all the possible relations between $k, p_{(k)}$, and $q_{(k)}$.

In the proof, we use obvious identities

$$
\left(\begin{array}{c}
n+1 \\
k
\end{array}\right)=\left(\begin{array}{l}
n \\
k
\end{array}\right)+\left(\begin{array}{c}
n \\
k-1
\end{array}\right)
$$

where $n, k \in \mathbb{N}$ and

$$
\begin{aligned}
& \left(\begin{array}{l}
i \\
i
\end{array}\right)=\left(\begin{array}{l}
i-1 \\
i-1
\end{array}\right), \quad\left(\begin{array}{l}
j \\
0
\end{array}\right)=\left(\begin{array}{c}
j-1 \\
0
\end{array}\right), \\
& \left(\begin{array}{c}
i+j \\
i
\end{array}\right)=\left(\begin{array}{c}
i+j-1 \\
i-1
\end{array}\right)+\left(\begin{array}{c}
i+j-1 \\
i
\end{array}\right),
\end{aligned}
$$

where $i, j \in \mathbb{N}$, derived from (4) and (24).

Now we consider (in parts (I)-(IV) below) all four cases and perform auxiliary computations. The proof will be finished in part (V).

(I) $\left(p_{(k)}-1\right)(m+1)+1 \leq k<p_{(k)}(m+1) \wedge\left(q_{(k)}-1\right)(n+1)+1 \leq$ $k<q_{(k)}(n+1)$. From (1) and (13), we get

$$
\begin{gathered}
p_{(k-m)}=\left\lfloor\frac{k-m+m}{m+1}\right\rfloor \leq \frac{k}{m+1}<p_{(k)}, \\
p_{(k-m)}=\left\lfloor\frac{k-m+m}{m+1}\right\rfloor>\frac{k}{m+1}-1=\frac{k-m-1}{m+1}>p_{(k)}-2 .
\end{gathered}
$$

Therefore, $p_{(k-m)}=p_{(k)}-1$ and, by Definition 6,

$$
\begin{aligned}
& \tilde{\mathrm{e}}_{m n}^{B C(k-m)} \\
& =I+B \sum_{i=0, j=0}^{P_{(k)}-2, q_{(k-m)}-1} B^{i} C^{j}\left(\begin{array}{c}
i+j \\
i
\end{array}\right)\left(\begin{array}{c}
k-m-m-m i-n j \\
i+j+1
\end{array}\right) \\
& \quad+C \sum_{i=0, j=0}^{P_{(k)}-2, q_{(k-m)}-1} B^{i} C^{j}\left(\begin{array}{c}
i+j \\
i
\end{array}\right)\left(\begin{array}{c}
k-m-n-m i-n j \\
i+j+1
\end{array}\right) .
\end{aligned}
$$

Similarly, omitting details, we get, using (1) and (13), $q_{(k-n)}=q_{(k)}-1$ and

$$
\begin{aligned}
& \widetilde{\mathrm{e}}_{m n}^{B C(k-n)} \\
& =I+B \sum_{i=0, j=0}^{p_{(k-n)}-1, q_{(k)}-2} B^{i} C^{j}\left(\begin{array}{c}
i+j \\
i
\end{array}\right)\left(\begin{array}{c}
k-n-m-m i-n j \\
i+j+1
\end{array}\right) \\
& \quad+C \sum_{i=0, j=0}^{p_{(k-n)}-1, q_{(k)}-2} B^{i} C^{j}\left(\begin{array}{c}
i+j \\
i
\end{array}\right)\left(\begin{array}{c}
k-n-n-m i-n j \\
i+j+1
\end{array}\right) .
\end{aligned}
$$

Let $q_{(k-m)} \geq 1$. We show that

$$
\begin{aligned}
& \left(\begin{array}{c}
k-m-m-m i-n j \\
i+j+1
\end{array}\right)=0 \quad \text { if } i \geq 0, \quad j \geq q_{(k-m)}, \\
& \left(\begin{array}{c}
k-m-n-m i-n j \\
i+j+1
\end{array}\right)=0 \quad \text { if } i \geq 0, \quad j \geq q_{(k-m)} .
\end{aligned}
$$

By (1),

$$
q_{(k-m)}=\left\lfloor\frac{k-m+n}{n+1}\right\rfloor>\frac{k-m+n}{n+1}-1=\frac{k-m-1}{n+1}
$$

or

$$
\begin{aligned}
k- & m-m \\
& <(n+1) q_{(k-m)}+1 \\
& \leq(m+1) i+(n+1) j+1 \quad \text { if } i \geq 0, j \geq q_{(k-m)}, \\
k- & m-n \\
& <(n+1) q_{(k-m)}+1 \\
& \leq(m+1) i+(n+1) j+1 \quad \text { if } i \geq 0, j \geq q_{(k-m)} .
\end{aligned}
$$

From the last inequalities, we get

$$
\begin{array}{ll}
k-m-m-m i-n j<i+j+1 & \text { if } i \geq 0, j \geq q_{(k-m)}, \\
k-m-n-m i-n j<i+j+1 & \text { if } i \geq 0, j \geq q_{(k-m)},
\end{array}
$$

and (29) holds by (4). For that reason and since $q_{(k-m)} \leq q_{(k)}$, we can replace $q_{(k-m)}$ by $q_{(k)}$ in (27). Thus, we have

$$
\begin{aligned}
& \widetilde{\mathrm{e}}_{m n}^{B C(k-m)} \\
& =I+B \sum_{i=0, j=0}^{p_{(k)}-2, q_{(k)}-1} B^{i} C^{j}\left(\begin{array}{c}
i+j \\
i
\end{array}\right)\left(\begin{array}{c}
k-m-m(i+1)-n j \\
i+j+1
\end{array}\right) \\
& \quad+C \sum_{i=0, j=0}^{p_{(k)}-2, q_{(k)}-1} B^{i} C^{j}\left(\begin{array}{c}
i+j \\
i
\end{array}\right)\left(\begin{array}{c}
k-n-m(i+1)-n j \\
i+j+1
\end{array}\right) .
\end{aligned}
$$


It is easy to see that, due to (5), formula (33) can be used instead of (27) if $q_{(k-m)}<1$ too. Let $p_{(k-n)} \geq 1$. Similarly, we can show that

$$
\begin{aligned}
& \left(\begin{array}{c}
k-n-m-m i-n j \\
i+j+1
\end{array}\right)=0 \quad \text { if } i \geq p_{(k-n)}, j \geq 0, \\
& \left(\begin{array}{c}
k-n-n-m i-n j \\
i+j+1
\end{array}\right)=0 \quad \text { if } i \geq p_{(k-n)}, j \geq 0,
\end{aligned}
$$

and, since $p_{(k-n)} \leq p_{(k)}$, we can replace $p_{(k-n)}$ by $p_{(k)}$ in (28). Thus, we have

$$
\begin{aligned}
& \widetilde{\mathrm{e}}_{m n}^{B C(k-n)} \\
& =I+B \sum_{i=0, j=0}^{p_{(k)}-1, q_{(k)}-2} B^{i} C^{j}\left(\begin{array}{c}
i+j \\
i
\end{array}\right)\left(\begin{array}{c}
k-m-m i-n(j+1) \\
i+j+1
\end{array}\right) \\
& \quad+C \sum_{i=0, j=0}^{p_{(k)}-1, q_{(k)}-2} B^{i} C^{j}\left(\begin{array}{c}
i+j \\
i
\end{array}\right)\left(\begin{array}{c}
k-n-m i-n(j+1) \\
i+j+1
\end{array}\right) .
\end{aligned}
$$

It is easy to see that, due to (5), formula (35) can be used instead of (28) if $p_{(k-n)}<1$ too By Definition 6,

$$
\begin{aligned}
& \widetilde{\mathrm{e}}_{m n}^{B C(k+1)} \\
& =I+B \sum_{i=0, j=0}^{p_{(k+1)}-1, q_{(k+1)}-1} B^{i} C^{j}\left(\begin{array}{c}
i+j \\
i
\end{array}\right)\left(\begin{array}{c}
k+1-m-m i-n j \\
i+j+1
\end{array}\right) \\
& \quad+C \sum_{i=0, j=0}^{p_{(k+1)}-1, q_{(k+1)}-1} B^{i} C^{j}\left(\begin{array}{c}
i+j \\
i
\end{array}\right)\left(\begin{array}{c}
k+1-n-m i-n j \\
i+j+1
\end{array}\right) .
\end{aligned}
$$

Due to (1), we also conclude that

$$
p_{(k+1)}=p_{(k)}, \quad q_{(k+1)}=q_{(k)}
$$

because

$$
\begin{aligned}
p_{(k+1)} & =\left\lfloor\frac{k+1+m}{m+1}\right\rfloor \leq \frac{k}{m+1}+1<p_{(k)}+1, \\
p_{(k+1)} & =\left\lfloor\frac{k+1+m}{m+1}\right\rfloor>\frac{k+1+m}{m+1}-1 \\
& =\frac{k}{m+1} \geq p_{(k)}-1+\frac{1}{m+1} .
\end{aligned}
$$

The second formula can be proved similarly.

Then,

$$
\begin{aligned}
& \widetilde{\mathrm{e}}_{m n}^{B C(k+1)} \\
& =I+B \sum_{i=0, j=0}^{p_{(k)}-1, q_{(k)}-1} B^{i} C^{j}\left(\begin{array}{c}
i+j \\
i
\end{array}\right)\left(\begin{array}{c}
k+1-m-m i-n j \\
i+j+1
\end{array}\right) \\
& \quad+C \sum_{i=0, j=0}^{p_{(k)}-1, q_{(k)}-1} B^{i} C^{j}\left(\begin{array}{c}
i+j \\
i
\end{array}\right)\left(\begin{array}{c}
k+1-n-m i-n j \\
i+j+1
\end{array}\right) .
\end{aligned}
$$

Now we are able to prove that

$$
\begin{aligned}
& \Delta \widetilde{\mathrm{e}}_{m n}^{B C k}=B \widetilde{\mathrm{e}}_{m n}^{B C(k-m)}+C \widetilde{\mathrm{e}}_{m n}^{B C(k-n)} \\
& =B\left[I+B \sum_{i=0, j=0}^{p_{(k)}-2, q_{(k)}-1} B^{i} C^{j}\left(\begin{array}{c}
i+j \\
i
\end{array}\right)\left(\begin{array}{c}
k-m-m(i+1)-n j \\
i+j+1
\end{array}\right)\right. \\
& \left.+C \sum_{i=0, j=0}^{p_{(k)}-2, q_{(k)}-1} B^{i} C^{j}\left(\begin{array}{c}
i+j \\
i
\end{array}\right)\left(\begin{array}{c}
k-n-m(i+1)-n j \\
i+j+1
\end{array}\right)\right] \\
& +C\left[I+B \sum_{i=0, j=0}^{p_{(k)}-1, q_{(k)}-2} B^{i} C^{j}\left(\begin{array}{c}
i+j \\
i
\end{array}\right)\left(\begin{array}{c}
k-m-m i-n(j+1) \\
i+j+1
\end{array}\right)\right. \\
& \left.+C \sum_{i=0, j=0}^{p_{(k)}-1, q_{(k)}-2} B^{i} C^{j}\left(\begin{array}{c}
i+j \\
i
\end{array}\right)\left(\begin{array}{c}
k-n-m i-n(j+1) \\
i+j+1
\end{array}\right)\right] .
\end{aligned}
$$

(II) $k=p_{(k)}(m+1) \wedge\left(q_{(k)}-1\right)(n+1)+1 \leq k<q_{(k)}(n+1)$. In this case,

$$
\begin{gathered}
p_{(k-m)}=\left\lfloor\frac{k-m+m}{m+1}\right\rfloor=\left\lfloor\frac{k}{m+1}\right\rfloor=p_{(k)}, \\
p_{(k+1)}=\left\lfloor\frac{k+1+m}{m+1}\right\rfloor \leq \frac{k+1+m}{m+1}=\frac{k}{m+1}+1=p_{(k)}+1, \\
p_{(k+1)}=\left\lfloor\frac{k+1+m}{m+1}\right\rfloor>\frac{k+1+m}{m+1}-1=\frac{k}{m+1}=p_{(k)}
\end{gathered}
$$

and $p_{(k+1)}=p_{(k)}+1$. In addition to this (see the relevant computations performed in case (I)), we have $q_{(k-n)}=q_{(k)}-1$ and $q_{(k+1)}=q_{(k)}$.

Then,

$$
\begin{aligned}
& \widetilde{\mathrm{e}}_{m n}^{B C(k+1)} \\
& =I+B \sum_{i=0, j=0}^{p_{(k)}, q_{(k)}-1} B^{i} C^{j}\left(\begin{array}{c}
i+j \\
i
\end{array}\right)\left(\begin{array}{c}
k+1-m-m i-n j \\
i+j+1
\end{array}\right) \\
& +C \sum_{i=0, j=0}^{p_{(k)}, q_{(k)}-1} B^{i} C^{j}\left(\begin{array}{c}
i+j \\
i
\end{array}\right)\left(\begin{array}{c}
k+1-n-m i-n j \\
i+j+1
\end{array}\right), \\
& \widetilde{\mathrm{e}}_{m n}^{B C(k-m)} \\
& =I+B \sum_{i=0, j=0}^{p_{(k)}-1, q_{(k-m)}-1} B^{i} C^{j}\left(\begin{array}{c}
i+j \\
i
\end{array}\right)\left(\begin{array}{c}
k-m-m-m i-n j \\
i+j+1
\end{array}\right) \\
& +C \sum_{i=0, j=0}^{p_{(k)}-1, q_{(k-m)}-1} B^{i} C^{j}\left(\begin{array}{c}
i+j \\
i
\end{array}\right)\left(\begin{array}{c}
k-m-n-m i-n j \\
i+j+1
\end{array}\right),
\end{aligned}
$$




$$
\begin{aligned}
& \mathrm{e}_{m n}^{B C(k-n)} \\
& =I+B \sum_{i=0, j=0}^{p_{(k-n)}^{-1, q_{(k)}-2}} B^{i} C^{j}\left(\begin{array}{c}
i+j \\
i
\end{array}\right)\left(\begin{array}{c}
k-n-m-m i-n j \\
i+j+1
\end{array}\right) \\
& +C \sum_{i=0, j=0}^{p_{(k-n)}-1, q_{(k)}-2} B^{i} C^{j}\left(\begin{array}{c}
i+j \\
i
\end{array}\right)\left(\begin{array}{c}
k-n-n-m i-n j \\
i+j+1
\end{array}\right) . \\
& \text { For } k=p_{(k)}(m+1), i=p_{(k)}, \text { and } j \geq 0, \text { we have } \\
& \left(\begin{array}{c}
k+1-m-m i-n j \\
i+j+1
\end{array}\right)=\left(\begin{array}{c}
p_{(k)}+1-m-n j \\
p_{(k)}+1+j
\end{array}\right)=0, \\
& \left(\begin{array}{c}
k+1-n-m i-n j \\
i+j+1
\end{array}\right)=\left(\begin{array}{c}
p_{(k)}+1-n-n j \\
p_{(k)}+1+j
\end{array}\right)=0
\end{aligned}
$$

and, for $k=p_{(k)}(m+1), i=p_{(k)}-1$, and $j \geq 0$, we have

$$
\begin{aligned}
& \left(\begin{array}{c}
k-m-m-m i-n j \\
i+j+1
\end{array}\right)=\left(\begin{array}{c}
p_{(k)}-m-n j \\
p_{(k)}+j
\end{array}\right)=0, \\
& \left(\begin{array}{c}
k-m-n-m i-n j \\
i+j+1
\end{array}\right)=\left(\begin{array}{c}
p_{(k)}-n-n j \\
p_{(k)}+j
\end{array}\right)=0 .
\end{aligned}
$$

Thus, we can substitute $p_{(k)}-1$ for $p_{(k)}$ in $(42)$ and $p_{(k)}-2$ for $p_{(k)}-1$ in (43).

Like with the computations performed in the previous part of the proof, (29), (34) hold. So we can substitute $q_{(k)}$ for $q_{(k-m)}$ in (43) and $p_{(k)}$ for $p_{(k-n)}$ in (44).

Accordingly, we have

$$
\begin{aligned}
& \widetilde{\mathrm{e}}_{m n}^{B C(k+1)} \\
& =I+B \sum_{i=0, j=0}^{p_{(k)}-1, q_{(k)}-1} B^{i} C^{j}\left(\begin{array}{c}
i+j \\
i
\end{array}\right)\left(\begin{array}{c}
k+1-m-m i-n j \\
i+j+1
\end{array}\right) \\
& +C \sum_{i=0, j=0}^{p_{(k)}-1, q_{(k)}-1} B^{i} C^{j}\left(\begin{array}{c}
i+j \\
i
\end{array}\right)\left(\begin{array}{c}
k+1-n-m i-n j \\
i+j+1
\end{array}\right), \\
& \widetilde{\mathrm{e}}_{m n}^{B C(k-m)} \\
& =I+B \sum_{i=0, j=0}^{p_{(k)}-2, q_{(k)}-1} B^{i} C^{j}\left(\begin{array}{c}
i+j \\
i
\end{array}\right)\left(\begin{array}{c}
k-m-m(i+1)-n j \\
i+j+1
\end{array}\right) \\
& +C \sum_{i=0, j=0}^{p_{(k)}-2, q_{(k)}-1} B^{i} C^{j}\left(\begin{array}{c}
i+j \\
i
\end{array}\right)\left(\begin{array}{c}
k-n-m(i+1)-n j \\
i+j+1
\end{array}\right),
\end{aligned}
$$

$$
\begin{aligned}
& \tilde{\mathrm{e}}_{m n}^{B C(k-n)} \\
& =I+B \sum_{i=0, j=0}^{p_{(k)}-1, q_{(k)}-2} B^{i} C^{j}\left(\begin{array}{c}
i+j \\
i
\end{array}\right)\left(\begin{array}{c}
k-m-m i-n(j+1) \\
i+j+1
\end{array}\right) \\
& \quad+C \sum_{i=0, j=0}^{p_{(k)}-1, q_{(k)}-2} B^{i} C^{j}\left(\begin{array}{c}
i+j \\
i
\end{array}\right)\left(\begin{array}{c}
k-n-m i-n(j+1) \\
i+j+1
\end{array}\right) .
\end{aligned}
$$

It is easy to see that, due to (5), formula (48) can also be used instead of (43) if $q_{(k-m)}<1$ and formula (49) can also be used instead of $(44)$ if $p_{(k-n)}<1$. Therefore, we see that (like in part (I)) the relation (40) must be proved.

(III) $\left(p_{(k)}-1\right)(m+1)+1 \leq k<p_{(k)}(m+1) \wedge k=q_{(k)}(n+1)$. In this case, we have (see the relevant computations in cases (I) and (II))

$$
\begin{aligned}
& p_{(k-m)}=p_{(k)}-1, \quad p_{(k+1)}=p_{(k)}, \\
& q_{(k-n)}=q_{(k)}, \quad q_{(k+1)}=q_{(k)}+1 .
\end{aligned}
$$

Then,

$$
\begin{aligned}
& \tilde{\mathrm{e}}_{m n}^{B C(k+1)} \\
& =I+B \sum_{i=0, j=0}^{p_{(k)}-1, q_{(k)}} B^{i} C^{j}\left(\begin{array}{c}
i+j \\
i
\end{array}\right)\left(\begin{array}{c}
k+1-m-m i-n j \\
i+j+1
\end{array}\right) \\
& \quad+C \sum_{i=0, j=0}^{p_{(k)}^{-1, q_{(k)}}} B^{i} C^{j}\left(\begin{array}{c}
i+j \\
i
\end{array}\right)\left(\begin{array}{c}
k+1-n-m i-n j \\
i+j+1
\end{array}\right),
\end{aligned}
$$

$\widetilde{\mathrm{e}}_{m n}^{B C(k-m)}$

$$
\begin{array}{r}
=I+B \sum_{i=0, j=0}^{p_{(k)}-2, q_{(k-m)}-1} B^{i} C^{j}\left(\begin{array}{c}
i+j \\
i
\end{array}\right)\left(\begin{array}{c}
k-m-m-m i-n j \\
i+j+1
\end{array}\right) \\
+C \sum_{i=0, j=0}^{p_{(k)}-2, q_{(k-m)}-1} B^{i} C^{j}\left(\begin{array}{c}
i+j \\
i
\end{array}\right)\left(\begin{array}{c}
k-m-n-m i-n j \\
i+j+1
\end{array}\right),
\end{array}
$$

$\widetilde{\mathrm{e}}_{m n}^{B C(k-n)}$

$$
\begin{gathered}
=I+B \sum_{i=0, j=0}^{p_{(k-n)}-1, q_{(k)}-1} B^{i} C^{j}\left(\begin{array}{c}
i+j \\
i
\end{array}\right)\left(\begin{array}{c}
k-n-m-m i-n j \\
i+j+1
\end{array}\right) \\
+C \sum_{i=0, j=0}^{p_{(k-n)}^{-1, q_{(k)}-1}} B^{i} C^{j}\left(\begin{array}{c}
i+j \\
i
\end{array}\right)\left(\begin{array}{c}
k-n-n-m i-n j \\
i+j+1
\end{array}\right) .
\end{gathered}
$$

For $k=q_{(k)}(n+1), j=q_{(k)}$, and $i \geq 0$, we have

$$
\begin{aligned}
& \left(\begin{array}{c}
k+1-m-m i-n j \\
i+j+1
\end{array}\right)=\left(\begin{array}{c}
q_{(k)}+1-m-m i \\
i+q_{(k)}+1
\end{array}\right)=0, \\
& \left(\begin{array}{c}
k+1-n-m i-n j \\
i+j+1
\end{array}\right)=\left(\begin{array}{c}
q_{(k)}+1-n-m i \\
i+q_{(k)}+1
\end{array}\right)=0
\end{aligned}
$$


and, for $k=q_{(k)}(m+1), j=q_{(k)}-1$, and $i \geq 0$, we get

$$
\begin{aligned}
& \left(\begin{array}{c}
k-n-m-m i-n j \\
i+j+1
\end{array}\right)=\left(\begin{array}{c}
q_{(k)}-m-m i \\
i+q_{(k)}
\end{array}\right)=0, \\
& \left(\begin{array}{c}
k-n-n-m i-n j \\
i+j+1
\end{array}\right)=\left(\begin{array}{c}
q_{(k)}-n-m i \\
i+q_{(k)}
\end{array}\right)=0 .
\end{aligned}
$$

Thus we can replace $q_{(k)}$ by $q_{(k)}-1$ in (51) and $q_{(k)}-1$ by $q_{(k)}-2$ in (53).

Like with the computations performed in cases (I) and (II), formulas (29), (34) hold and we can substitute $q_{(k)}$ for $q_{(k-m)}$ in (52) and $q_{(k)}$ for $q_{(k-n)}$ in (53). This means that

$$
\begin{aligned}
& \widetilde{\mathrm{e}}_{m n}^{B C(k+1)} \\
& =I+B \sum_{i=0, j=0}^{p_{(k)}-1, q_{(k)}-1} B^{i} C^{j}\left(\begin{array}{c}
i+j \\
i
\end{array}\right)\left(\begin{array}{c}
k+1-m-m i-n j \\
i+j+1
\end{array}\right) \\
& +C \sum_{i=0, j=0}^{p_{(k)}-1, q_{(k)}-1} B^{i} C^{j}\left(\begin{array}{c}
i+j \\
i
\end{array}\right)\left(\begin{array}{c}
k+1-n-m i-n j \\
i+j+1
\end{array}\right), \\
& \widetilde{\mathrm{e}}_{m n}^{B C(k-m)} \\
& =I+B \sum_{i=0, j=0}^{p_{(k)}-2, q_{(k-m)}-1} B^{i} C^{j}\left(\begin{array}{c}
i+j \\
i
\end{array}\right)\left(\begin{array}{c}
k-m-m-m i-n j \\
i+j+1
\end{array}\right) \\
& +C \sum_{i=0, j=0}^{p_{(k)}-2, q_{(k-m)}-1} B^{i} C^{j}\left(\begin{array}{c}
i+j \\
i
\end{array}\right)\left(\begin{array}{c}
k-m-n-m i-n j \\
i+j+1
\end{array}\right), \\
& =I+B \sum_{i=0, j=0}^{p_{(k-n)}-1, q_{(k)}-2} B^{i} C^{j}\left(\begin{array}{c}
i+j \\
i
\end{array}\right)\left(\begin{array}{c}
k-n-m-m i-n j \\
i+j+1
\end{array}\right) \\
& +C \sum_{i=0, j=0}^{p_{(k-n)}^{-1, q_{(k)}-2}} B^{i} C^{j}\left(\begin{array}{c}
i+j \\
i
\end{array}\right)\left(\begin{array}{c}
k-n-n-m i-n j \\
i+j+1
\end{array}\right) .
\end{aligned}
$$

It is easy to see that, due to (5), formula (57) can also be used instead of (52) if $q_{(k-m)}<1$ and formula (58) can also be used instead of (53) if $p_{(k-n)}<1$. Therefore, we see that (as in parts (I), (II)) (40) must be proved.

(IV) $k=p_{(\mathrm{k})}(m+1) \wedge k=q_{(k)}(n+1)$. In this case, we have (see similar combinations in the cases (II) and (III))

$$
\begin{array}{ll}
p_{(k-m)}=p_{(k)}, & p_{(k+1)}=p_{(k)}+1, \\
q_{(k-n)}=q_{(k)}, & q_{(k+1)}=q_{(k)}+1 .
\end{array}
$$

Then,

$$
\begin{aligned}
& \widetilde{\mathrm{e}}_{m n}^{B C(k+1)} \\
& =I+B \sum_{i=0, j=0}^{p_{(k)}, q_{(k)}} B^{i} C^{j}\left(\begin{array}{c}
i+j \\
i
\end{array}\right)\left(\begin{array}{c}
k+1-m-m i-n j \\
i+j+1
\end{array}\right) \\
& +C \sum_{i=0, j=0}^{p_{(k)}, q_{(k)}} B^{i} C^{j}\left(\begin{array}{c}
i+j \\
i
\end{array}\right)\left(\begin{array}{c}
k+1-n-m i-n j \\
i+j+1
\end{array}\right), \\
& \widetilde{\mathrm{e}}_{m n}^{B C(k-m)} \\
& =I+B \sum_{i=0, j=0}^{p_{(k)}-1, q_{(k-m)}-1} B^{i} C^{j}\left(\begin{array}{c}
i+j \\
i
\end{array}\right)\left(\begin{array}{c}
k-m-m-m i-n j \\
i+j+1
\end{array}\right) \\
& +C \sum_{i=0, j=0}^{p_{(k)}-1, q_{(k-m)}-1} B^{i} C^{j}\left(\begin{array}{c}
i+j \\
i
\end{array}\right)\left(\begin{array}{c}
k-m-n-m i-n j \\
i+j+1
\end{array}\right), \\
& \widetilde{\mathrm{e}}_{m n}^{B C(k-n)} \\
& =I+B \sum_{i=0, j=0}^{p_{(k-n)}-1, q_{(k)}-1} B^{i} C^{j}\left(\begin{array}{c}
i+j \\
i
\end{array}\right)\left(\begin{array}{c}
k-n-m-m i-n j \\
i+j+1
\end{array}\right) \\
& +C \sum_{i=0, j=0}^{p_{(k-n)}-1, q_{(k)}-1} B^{i} C^{j}\left(\begin{array}{c}
i+j \\
i
\end{array}\right)\left(\begin{array}{c}
k-n-n-m i-n j \\
i+j+1
\end{array}\right) .
\end{aligned}
$$

As in part (II), for $k=p_{(k)}(m+1), i=p_{(k)}$, and $j \geq 0$, formulas (45) hold and, for $k=p_{(k)}(m+1), i=p_{(k)}-1$, and $j \geq 0$, formulas (46) hold. Thus we can substitute $p_{(k)}-1$ for $p_{(k)}$ in (60) and $p_{(k)}-2$ for $p_{(k)}-1$ in (61).

As in part (III), for $k=q_{(k)}(n+1), j=q_{(k)}$, and $i \geq 0$, formulas (54) hold and, for $k=q_{(k)}(m+1), j=q_{(k)}-1$, and $i \geq 0$, formulas (55) hold. Thus we can replace $q_{(k)}$ by $q_{(k)}-1$ in $(60)$ and $q_{(k)}-1$ by $q_{(k)}-2$ in (62).

As before, (29), (34) hold and we can substitute $q_{(k)}$ for $q_{(k-m)}$ in (61) and $p_{(k)}$ for $p_{(k-n)}$ in (62). Thus, we have

$$
\begin{aligned}
& \widetilde{\mathrm{e}}_{m n}^{B C(k+1)} \\
& =I+B \sum_{i=0, j=0}^{p_{(k)}-1, q_{(k)}-1} B^{i} C^{j}\left(\begin{array}{c}
i+j \\
i
\end{array}\right)\left(\begin{array}{c}
k+1-m-m i-n j \\
i+j+1
\end{array}\right) \\
& \quad+C \sum_{i=0, j=0}^{p_{(k)}-1, q_{(k)}-1} B^{i} C^{j}\left(\begin{array}{c}
i+j \\
i
\end{array}\right)\left(\begin{array}{c}
k+1-n-m i-n j \\
i+j+1
\end{array}\right),
\end{aligned}
$$




$$
\begin{aligned}
& \widetilde{\mathrm{e}}_{m n}^{B C(k-m)} \\
& =I+B \sum_{i=0, j=0}^{p_{(k)}-2, q_{(k-m)}-1} B^{i} C^{j}\left(\begin{array}{c}
i+j \\
i
\end{array}\right)\left(\begin{array}{c}
k-m-m-m i-n j \\
i+j+1
\end{array}\right) \\
& +C \sum_{i=0, j=0}^{p_{(k)}-2, q_{(k-m)}-1} B^{i} C^{j}\left(\begin{array}{c}
i+j \\
i
\end{array}\right)\left(\begin{array}{c}
k-m-n-m i-n j \\
i+j+1
\end{array}\right), \\
& \widetilde{\mathrm{e}}_{m n}^{B C(k-n)} \\
& =I+B \sum_{i=0, j=0}^{p_{(k-n)}-1, q_{(k)}-2} B^{i} C^{j}\left(\begin{array}{c}
i+j \\
i
\end{array}\right)\left(\begin{array}{c}
k-n-m-m i-n j \\
i+j+1
\end{array}\right) \\
& +C \sum_{i=0, j=0}^{p_{(k-n)}-1, q_{(k)}-2} B^{i} C^{j}\left(\begin{array}{c}
i+j \\
i
\end{array}\right)\left(\begin{array}{c}
k-n-n-m i-n j \\
i+j+1
\end{array}\right) .
\end{aligned}
$$

It is easy to see that, due to (5), formula (64) can also be used instead of (61) if $q_{(k-m)}<1$ and formula (65) can also be used instead of $(62)$ if $p_{(k-n)}<1$. Therefore, we see that (as in all the previous parts) (40) must be proved.

(V) The Proof of Formula (40). Now we prove (40). With the aid of (18), (19), (24), and (36), we get

$$
\begin{aligned}
& \Delta \widetilde{\mathrm{e}}_{m n}^{B C k} \\
& =\widetilde{\mathrm{e}}_{m n}^{B C(k+1)}-\widetilde{\mathrm{e}}_{m n}^{B C k} \\
& =I+B \sum_{i=0, j=0}^{p_{(k)}-1, q_{(k)}-1} B^{i} C^{j}\left(\begin{array}{c}
i+j \\
i
\end{array}\right)\left(\begin{array}{c}
k+1-m-m i-n j \\
i+j+1
\end{array}\right) \\
& +C \sum_{i=0, j=0}^{p_{(k)}-1, q_{(k)}-1} B^{i} C^{j}\left(\begin{array}{c}
i+j \\
i
\end{array}\right)\left(\begin{array}{c}
k+1-n-m i-n j \\
i+j+1
\end{array}\right) \\
& -I-B \sum_{i=0, j=0}^{p_{(k)}-1, q_{(k)}-1} B^{i} C^{j}\left(\begin{array}{c}
i+j \\
i
\end{array}\right)\left(\begin{array}{c}
k-m-m i-n j \\
i+j+1
\end{array}\right) \\
& -C \sum_{i=0, j=0}^{p_{(k)}-1, q_{(k)}-1} B^{i} C^{j}\left(\begin{array}{c}
i+j \\
i
\end{array}\right)\left(\begin{array}{c}
k-n-m i-n j \\
i+j+1
\end{array}\right) \\
& =B \sum_{i=0, j=0}^{p_{(k)}-1, q_{(k)}-1} B^{i} C^{j}\left(\begin{array}{c}
i+j \\
i
\end{array}\right)\left[\left(\begin{array}{c}
k+1-m-m i-n j \\
i+j+1
\end{array}\right)\right. \\
& \left.-\left(\begin{array}{c}
k-m-m i-n j \\
i+j+1
\end{array}\right)\right] \\
& +C \sum_{i=0, j=0}^{p_{(k)}-1, q_{(k)}-1} B^{i} C^{j}\left(\begin{array}{c}
i+j \\
i
\end{array}\right)\left[\left(\begin{array}{c}
k+1-n-m i-n j \\
i+j+1
\end{array}\right)\right. \\
& \left.-\left(\begin{array}{c}
k-n-m i-n j \\
i+j+1
\end{array}\right)\right]
\end{aligned}
$$

$$
\begin{aligned}
& =B \sum_{i=0, j=0}^{p_{(k)}-1, q_{(k)}-1} B^{i} C^{j}\left(\begin{array}{c}
i+j \\
i
\end{array}\right)\left(\begin{array}{c}
k-m-m \mathbf{i}-n j \\
i+j
\end{array}\right) \\
& +C \sum_{i=0, j=0}^{p_{(k)}-1, q_{(k)}-1} B^{i} C^{j}\left(\begin{array}{c}
i+j \\
i
\end{array}\right)\left(\begin{array}{c}
k-n-m i-n j \\
i+j
\end{array}\right) \\
& =B\left[I+\sum_{i=1}^{p_{(k)}-1} B^{i} C^{0}\left(\begin{array}{c}
i \\
i
\end{array}\right)\left(\begin{array}{c}
k-m-m i \\
i
\end{array}\right)\right. \\
& +\sum_{j=1}^{q_{(k)}-1} B^{0} C^{j}\left(\begin{array}{l}
j \\
0
\end{array}\right)\left(\begin{array}{c}
k-m-n j \\
j
\end{array}\right) \\
& \left.+\sum_{i=1, j=1}^{p_{(k)}-1, q_{(k)}-1} B^{i} C^{j}\left(\begin{array}{c}
i+j \\
i
\end{array}\right)\left(\begin{array}{c}
k-m-m i-n j \\
i+j
\end{array}\right)\right] \\
& +C\left[I+\sum_{i=1}^{p_{(k)}-1} B^{i} C^{0}\left(\begin{array}{l}
i \\
i
\end{array}\right)\left(\begin{array}{c}
k-n-m i \\
i
\end{array}\right)\right. \\
& +\sum_{j=1}^{q_{(k)}-1} B^{0} C^{j}\left(\begin{array}{l}
j \\
0
\end{array}\right)\left(\begin{array}{c}
k-n-n j \\
j
\end{array}\right) \\
& \left.+\sum_{i=1, j=1}^{p_{(k)}-1, q_{(k)}-1} B^{i} C^{j}\left(\begin{array}{c}
i+j \\
i
\end{array}\right)\left(\begin{array}{c}
k-n-m i-n j \\
i+j
\end{array}\right)\right] .
\end{aligned}
$$

By (25), we have

$$
\begin{aligned}
& \Delta \widetilde{\mathrm{e}}_{m n}^{B C k} \\
& =B\left[I+\sum_{i=1}^{p_{(k)}-1} B^{i} C^{0}\left(\begin{array}{c}
i-1 \\
i-1
\end{array}\right)\left(\begin{array}{c}
k-m-m i \\
i
\end{array}\right)\right. \\
& +\sum_{j=1}^{q_{(k)}-1} B^{0} C^{j}\left(\begin{array}{c}
j-1 \\
0
\end{array}\right)\left(\begin{array}{c}
k-m-n j \\
j
\end{array}\right) \\
& +\sum_{i=1, j=1}^{p_{(k)}-1, q_{(k)}-1} B^{i} C^{j}\left(\begin{array}{c}
i+j-1 \\
i-1
\end{array}\right)\left(\begin{array}{c}
k-m-m i-n j \\
i+j
\end{array}\right) \\
& \left.+\sum_{i=1, j=1}^{p_{(k)}-1, q_{(k)}-1} B^{i} C^{j}\left(\begin{array}{c}
i+j-1 \\
i
\end{array}\right)\left(\begin{array}{c}
k-m-m i-n j \\
i+j
\end{array}\right)\right] \\
& +C\left[I+\sum_{i=1}^{p_{(k)}-1} B^{i} C^{0}\left(\begin{array}{c}
i-1 \\
i-1
\end{array}\right)\left(\begin{array}{c}
k-n-m i \\
i
\end{array}\right)\right. \\
& +\sum_{j=1}^{q_{(\mathrm{k})}-1} B^{0} C^{j}\left(\begin{array}{c}
j-1 \\
0
\end{array}\right)\left(\begin{array}{c}
k-n-n j \\
j
\end{array}\right) \\
& +\sum_{i=1, j=1}^{p_{(k)}-1, q_{(k)}-1} B^{i} C^{j}\left(\begin{array}{c}
i+j-1 \\
i-1
\end{array}\right)\left(\begin{array}{c}
k-n-m i-n j \\
i+j
\end{array}\right)
\end{aligned}
$$




$$
\begin{array}{r}
\left.+\sum_{i=1, j=1}^{p_{(k)}-1, q_{(k)}-1} B^{i} C^{j}\left(\begin{array}{c}
i+j-1 \\
i
\end{array}\right)\left(\begin{array}{c}
k-n-m i-n j \\
i+j
\end{array}\right)\right] \\
=B\left[I+\sum_{i=1, j=0}^{p_{(k)}-1, q_{(k)}-1} B^{i} C^{j}\left(\begin{array}{c}
i+j-1 \\
i-1
\end{array}\right)\left(\begin{array}{c}
k-m-m i-n j \\
i+j
\end{array}\right)\right. \\
\left.+\sum_{i=0, j=1}^{p_{(k)}-1, q_{(k)}-1} B^{i} C^{j}\left(\begin{array}{c}
i+j-1 \\
i
\end{array}\right)\left(\begin{array}{c}
k-m-m i-n j \\
i+j
\end{array}\right)\right] \\
+\left[\begin{array}{c}
I+\sum_{i=1, j=0}^{p_{(k)}-1, q_{(k)}-1} B^{i} C^{j}\left(\begin{array}{c}
i+j-1 \\
i-1
\end{array}\right)\left(\begin{array}{c}
k-n-m i-n j \\
i+j
\end{array}\right) \\
\left.+\sum_{i=0, j=1}^{p_{(k)}-1, q_{(k)}-1} B^{i} C^{j}\left(\begin{array}{c}
i+j-1 \\
i
\end{array}\right)\left(\begin{array}{c}
k-n-m i-n j \\
i+j
\end{array}\right)\right] .
\end{array}\right.
\end{array}
$$

Now, in the first and third sum, we replace the summation index $i$ by $i+1$ and, in the second and fourth sum, we replace the summation index $j$ by $j+1$. Then,

$\Delta \widetilde{\mathrm{e}}_{m n}^{B C k}$

$$
\begin{aligned}
& =B\left[I+\sum_{i=0, j=0}^{p_{(k)}-2, q_{(k)}-1} B^{i+1} C^{j}\left(\begin{array}{c}
i+j \\
i
\end{array}\right)\right. \\
& \times\left(\begin{array}{c}
k-m-m(i+1)-n j \\
i+j+1
\end{array}\right) \\
& \left.+\sum_{i=0, j=0}^{p_{(k)}-1, q_{(k)}-2} B^{i} C^{j+1}\left(\begin{array}{c}
i+j \\
i
\end{array}\right)\left(\begin{array}{c}
k-m-m i-n(j+1) \\
i+j+1
\end{array}\right)\right] \\
& +C\left[I+\sum_{i=0, j=0}^{p_{(k)}-2, q_{(k)}-1} B^{i+1} C^{j}\left(\begin{array}{c}
i+j \\
i
\end{array}\right)\right. \\
& \times\left(\begin{array}{c}
k-n-m(i+1)-n j \\
i+j+1
\end{array}\right) \\
& \left.+\sum_{i=0, j=0}^{p_{(k)}-1, q_{(k)}-2} B^{i} C^{j+1}\left(\begin{array}{c}
i+j \\
i
\end{array}\right)\left(\begin{array}{c}
k-n-m i-n(j+1) \\
i+j+1
\end{array}\right)\right] \\
& =B+B^{2} \sum_{i=0, j=0}^{p_{(k)}-2, q_{(k)}-1} B^{i} C^{j}\left(\begin{array}{c}
i+j \\
i
\end{array}\right)\left(\begin{array}{c}
k-m-m(i+1)-n j \\
i+j+1
\end{array}\right) \\
& +B C \sum_{i=0, j=0}^{p_{(k)}-1, q_{(k)}-2} B^{i} C^{j}\left(\begin{array}{c}
i+j \\
i
\end{array}\right)\left(\begin{array}{c}
k-m-m i-n(j+1) \\
i+j+1
\end{array}\right) \\
& +C+B C \sum_{i=0, j=0}^{p_{(k)}-2, q_{(k)}-1} B^{i} C^{j}\left(\begin{array}{c}
i+j \\
i
\end{array}\right) \\
& \times\left(\begin{array}{c}
k-n-m(i+1)-n j \\
i+j+1
\end{array}\right)
\end{aligned}
$$

$$
\begin{aligned}
& +C^{2} \sum_{i=0, j=0}^{p_{(k)}-1, q_{(k)}-2} B^{i} C^{j}\left(\begin{array}{c}
i+j \\
i
\end{array}\right)\left(\begin{array}{c}
k-n-m i-n(j+1) \\
i+j+1
\end{array}\right) \\
& =B\left[I+B \sum_{i=0, j=0}^{p_{(k)}-2, q_{(k)}-1} B^{i} C^{j}\left(\begin{array}{c}
i+j \\
i
\end{array}\right)\left(\begin{array}{c}
k-m-m(i+1)-n j \\
i+j+1
\end{array}\right)\right. \\
& \left.+C \sum_{i=0, j=0}^{p_{(k)}-2, q_{(k)}-1} B^{i} C^{j}\left(\begin{array}{c}
i+j \\
i
\end{array}\right)\left(\begin{array}{c}
k-n-m(i+1)-n j \\
i+j+1
\end{array}\right)\right] \\
& +C\left[I+B \sum_{i=0, j=0}^{p_{(k)}-1, q_{(k)}-2} B^{i} C^{j}\left(\begin{array}{c}
i+j \\
i
\end{array}\right)\right. \\
& \times\left(\begin{array}{c}
k-m-m i-n(j+1) \\
i+j+1
\end{array}\right) \\
& \left.+C \sum_{i=0, j=0}^{p_{(k)}-1, q_{(k)}-2} B^{i} C^{j}\left(\begin{array}{c}
i+j \\
i
\end{array}\right)\left(\begin{array}{c}
k-n-m i-n(j+1) \\
i+j+1
\end{array}\right)\right] \\
& =B \widetilde{\mathrm{e}}_{m n}^{B C(k-m)}+C \widetilde{\mathrm{e}}_{m n}^{B C(k-n)} .
\end{aligned}
$$

Due to (33) and (35), we conclude that formula (40) is valid.

We proved that formula (15) holds in each of the considered cases (I), (II), (III), and (IV) for $k \geq 1$. If $k=0$, the proof can be done directly because $p_{(0)}=q_{(0)}=0, p_{(1)}=q_{(1)}=1$,

$$
\begin{aligned}
\Delta \widetilde{\mathrm{e}}_{m n}^{B C 0}= & \widetilde{\mathrm{e}}_{m n}^{B C 1}-\widetilde{\mathrm{e}}_{m n}^{B C 0} \\
= & +B \sum_{i=0, j=0}^{0,0} B^{i} C^{j}\left(\begin{array}{c}
i+j \\
i
\end{array}\right)\left(\begin{array}{c}
1-m-m i-n j \\
i+j+1
\end{array}\right) \\
& +C \sum_{i=0, j=0}^{0,0} B^{i} C^{j}\left(\begin{array}{c}
i+j \\
i
\end{array}\right)\left(\begin{array}{c}
1-n-m i-n j \\
i+j+1
\end{array}\right) \\
& -I-B \sum_{i=0, j=0}^{-1,-1} B^{i} C^{j}\left(\begin{array}{c}
i+j \\
i
\end{array}\right)\left(\begin{array}{c}
-m-m i-n j \\
i+j+1
\end{array}\right) \\
& -C \sum_{i=0, j=0}^{-1,-1} B^{i} C^{j}\left(\begin{array}{c}
i+j \\
i
\end{array}\right)\left(\begin{array}{c}
-n-m i-n j \\
i+j+1
\end{array}\right) \\
= & I+B \Theta+C \Theta-I-B \Theta-C \Theta=\Theta, \\
& B \widetilde{\mathrm{e}}_{m n}^{B C(-m)}+C \widetilde{\mathrm{e}}_{m n}^{B C(-n)}=B \Theta+C \Theta=\Theta .
\end{aligned}
$$

Formula (15) holds again. Theorem 9 is proved.

\section{Representing the Solution of an Initial Problem by Discrete Matrix Delayed Exponential for Two Delays}

In this part, we prove the main results of the paper. With the aid of both discrete matrix delayed exponentials we 
give formulas for the solution of the homogeneous and nonhomogeneous initial problem (2), (3).

3.1. Representing the Solution of a Homogeneous Initial Problem. Consider the homogeneous initial problem

$$
\begin{gathered}
\Delta x(k)=B x(k-m)+C x(k-n), \quad k \in \mathbb{Z}_{0}^{\infty}, \\
x(k)=\varphi(k), \quad k \in \mathbb{Z}_{-n^{0}}^{0}
\end{gathered}
$$

First we derive formulas for the solution of (70), (71) with the aid of discrete matrix delayed exponential $\mathrm{e}_{m n}^{B C k}$ and then with the aid of discrete matrix delayed exponential $\widetilde{\mathrm{e}}_{m n}^{B C k}$.

Theorem 10. Let $B, C$ be constant $r \times r$ matrices such that

$$
B C=C B, \quad \operatorname{det}(B+C) \neq 0,
$$

and let $m, n \in \mathbb{N}, m<n$, be fixed integers. Then, the solution of the initial Cauchy problem (70), (71) can be expressed in the form

$$
x(k)=\sum_{j=0}^{n} e_{m n}^{B C(k+j)} v_{j},
$$

where $k \in \mathbb{Z}_{-n}^{\infty}$ and

$$
\begin{gathered}
v_{0}=\varphi(-n)-\sum_{s=1}^{n} v_{s}, \\
v_{\ell}=(B+C)^{-1}\left[\Delta \varphi(-\ell)-\sum_{t=1}^{n-\ell} \Delta e_{m n}^{B C t} v_{t+\ell}\right], \quad \ell \in \mathbb{Z}_{1}^{n} .
\end{gathered}
$$

Proof. We are going to find the solution of the problem (70), (71) in the form

$$
x(k)=\sum_{j=0}^{n} \mathrm{e}_{m n}^{B C(k+j)} v_{j}, \quad k \in \mathbb{Z}_{-n}^{\infty}
$$

with unknown constant vectors $v_{j}$. Due to linearity (taking into account that $k$ varies), we have, for $k \geq 0$,

$$
\begin{aligned}
\Delta x(k) & =\Delta \sum_{j=0}^{n} \mathrm{e}_{m n}^{B C(k+j)} v_{j}=\sum_{j=0}^{n} \Delta\left[\mathrm{e}_{m n}^{B C(k+j)} v_{j}\right] \\
& =\sum_{j=0}^{n} \Delta\left[\mathrm{e}_{m n}^{B C(k+j)}\right] v_{j} .
\end{aligned}
$$

Using formula (11),

$$
\begin{aligned}
\Delta x(k) & =\sum_{j=0}^{n}\left(B \mathrm{e}_{m n}^{B C(k-m+j)}+C \mathrm{e}_{m n}^{B C(k-n+j)}\right) v_{j} \\
& =B \sum_{j=0}^{n} \mathrm{e}_{m n}^{B C(k-m+j)} v_{j}+C \sum_{j=0}^{n} \mathrm{e}_{m n}^{B C(k-n+j)} v_{j} \\
& =B x(k-m)+C x(k-n) .
\end{aligned}
$$

Now we conclude that, for any $v_{j}$ and $k \in \mathbb{Z}_{0}^{\infty}$, the equation $\Delta x(k)=B x(k-m)+C x(k-n)$ holds. We will try to satisfy initial conditions (71). Due to (75), we have, for $k \in \mathbb{Z}_{-n}^{0}$,

$$
\begin{aligned}
& \mathrm{e}_{m n}^{B C 0} v_{0}+\mathrm{e}_{m n}^{B C 1} v_{1}+\mathrm{e}_{m n}^{B C 2} v_{2}+\cdots+\mathrm{e}_{m n}^{B C(n-2)} v_{n-2} \\
& +\mathrm{e}_{m n}^{B C(n-1)} v_{n-1}+\mathrm{e}_{m n}^{B C n} v_{n}=\varphi(0), \\
& \mathrm{e}_{m n}^{B C(-1)} v_{0}+\mathrm{e}_{m n}^{B C 0} v_{1}+\mathrm{e}_{m n}^{B C 1} v_{2}+\cdots+\mathrm{e}_{m n}^{B C(n-3)} v_{n-2} \\
& +\mathrm{e}_{m n}^{B C(n-2)} v_{n-1}+\mathrm{e}_{m n}^{B C(n-1)} v_{n}=\varphi(-1), \\
& \mathrm{e}_{m n}^{B C(-2)} v_{0}+\mathrm{e}_{m n}^{B C(-1)} v_{1}+\mathrm{e}_{m n}^{B C 0} v_{2}+\cdots+\mathrm{e}_{m n}^{B C(n-4)} v_{n-2} \\
& +\mathrm{e}_{m n}^{B C(n-3)} v_{n-1}+\mathrm{e}_{m n}^{B C(n-2)} v_{n}=\varphi(-2), \\
& \mathrm{e}_{m n}^{B C(-3)} v_{0}+\mathrm{e}_{m n}^{B C(-2)} v_{1}+\mathrm{e}_{m n}^{B C(-1)} v_{2}+\cdots+\mathrm{e}_{m n}^{B C(n-5)} v_{n-2} \\
& +\mathrm{e}_{m n}^{B C(n-4)} v_{n-1}+\mathrm{e}_{m n}^{B C(n-3)} v_{n}=\varphi(-3), \\
& \mathrm{e}_{m n}^{B C(-n+3)} v_{0}+\mathrm{e}_{m n}^{B C(-n+4)} v_{1}+\mathrm{e}_{m n}^{B C(-n+5)} v_{2}+\cdots+\mathrm{e}_{m n}^{B C 1} v_{n-2} \\
& +\mathrm{e}_{m n}^{B C 2} v_{n-1}+\mathrm{e}_{m n}^{B C 3} v_{n}=\varphi(-n+3), \\
& \mathrm{e}_{m n}^{B C(-n+2)} v_{0}+\mathrm{e}_{m n}^{B C(-n+3)} v_{1}+\mathrm{e}_{m n}^{B C(-n+4)} v_{2}+\cdots+\mathrm{e}_{m n}^{B C 0} v_{n-2} \\
& +\mathrm{e}_{m n}^{B C 1} v_{n-1}+\mathrm{e}_{m n}^{B C 2} v_{n}=\varphi(-n+2), \\
& \mathrm{e}_{m n}^{B C(-n+1)} v_{0}+\mathrm{e}_{m n}^{B C(-n+2)} v_{1}+\mathrm{e}_{m n}^{B C(-n+3)} v_{2}+\cdots+\mathrm{e}_{m n}^{B C(-1)} v_{n-2} \\
& +\mathrm{e}_{m n}^{B C 0} v_{n-1}+\mathrm{e}_{m n}^{B C 1} v_{n}=\varphi(-n+1), \\
& \mathrm{e}_{m n}^{B C(-n)} v_{0}+\mathrm{e}_{m n}^{B C(-n+1)} v_{1}+\mathrm{e}_{m n}^{B C(-n+2)} v_{2}+\cdots+\mathrm{e}_{m n}^{B C(-2)} v_{n-2} \\
& +\mathrm{e}_{m n}^{B C(-1)} v_{n-1}+\mathrm{e}_{m n}^{B C 0} v_{n}=\varphi(-n) .
\end{aligned}
$$

Due to Definition 3, $\mathrm{e}_{m n}^{B C k}=I$ for $k \in \mathbb{Z}_{-n}^{0}$. So we have

$$
\begin{gathered}
v_{0}+\mathrm{e}_{m n}^{B C 1} v_{1}+\mathrm{e}_{m n}^{B C 2} v_{2}+\cdots+\mathrm{e}_{m n}^{B C(n-2)} v_{n-2} \\
+\mathrm{e}_{m n}^{B C(n-1)} v_{n-1}+\mathrm{e}_{m n}^{B C n} v_{n}=\varphi(0), \\
v_{0}+v_{1}+\mathrm{e}_{m n}^{B C 1} v_{2}+\cdots+\mathrm{e}_{m n}^{B C(n-3)} v_{n-2} \\
+\mathrm{e}_{m n}^{B C(n-2)} v_{n-1}+\mathrm{e}_{m n}^{B C(n-1)} v_{n}=\varphi(-1), \\
v_{0}+v_{1}+v_{2}+\cdots+\mathrm{e}_{m n}^{B C(n-4)} v_{n-2} \\
+\mathrm{e}_{m n}^{B C(n-3)} v_{n-1}+\mathrm{e}_{m n}^{B C(n-2)} v_{n}=\varphi(-2), \\
v_{0}+v_{1}+v_{2}+\cdots+\mathrm{e}_{m n}^{B C(n-5)} v_{n-2} \\
+\mathrm{e}_{m n}^{B C(n-4)} v_{n-1}+\mathrm{e}_{m n}^{B C(n-3)} v_{n}=\varphi(-3),
\end{gathered}
$$




$$
\begin{aligned}
& v_{0}+ v_{1}+v_{2}+\cdots+\mathrm{e}_{m n}^{B C 1} v_{n-2} \\
&+\mathrm{e}_{m n}^{B C 2} v_{n-1}+\mathrm{e}_{m n}^{B C 3} v_{n}=\varphi(-n+3) \\
& v_{0}+v_{1}+v_{2}+\cdots+v_{n-2} \\
&+\mathrm{e}_{m n}^{B C 1} v_{n-1}+\mathrm{e}_{m n}^{B C 2} v_{n}=\varphi(-n+2) \\
& v_{0}+ v_{1}+v_{2}+\cdots+v_{n-2} \\
&+v_{n-1}+\mathrm{e}_{m n}^{B C 1} v_{n}=\varphi(-n+1) \\
& v_{0}+v_{1}+v_{2}+\cdots+v_{n-2}+v_{n-1}+v_{n}=\varphi(-n)
\end{aligned}
$$

Subtracting the neighbouring equations $\left(\left(E_{n-1}-E_{n}\right)\right.$, $\left.\left(E_{n-2}-E_{n-1}\right), \ldots,\left(E_{0}-E_{1}\right)\right)$, we get

$$
\begin{aligned}
& \left(\mathrm{e}_{m n}^{B C 1}-I\right) v_{n}=\varphi(-n+1)-\varphi(-n), \quad\left(E_{n-1}-E_{n}\right) \\
& \left(\mathrm{e}_{m n}^{B C 1}-I\right) v_{n-1}+\left(\mathrm{e}_{m n}^{B C 2}-\mathrm{e}_{m n}^{B C 1}\right) v_{n} \\
& =\varphi(-n+2)-\varphi(-n+1) \text {, } \\
& \left(E_{n-2}-E_{n-1}\right) \\
& \left(\mathrm{e}_{m n}^{B C 1}-I\right) v_{n-2}+\left(\mathrm{e}_{m n}^{B C 2}-\mathrm{e}_{m n}^{B C 1}\right) v_{n-1}+\left(\mathrm{e}_{m n}^{B C 3}-\mathrm{e}_{m n}^{B C 2}\right) v_{n} \\
& =\varphi(-n+3)-\varphi(-n+2) \text {, } \\
& \left(E_{n-3}-E_{n-2}\right) \\
& \left(\mathrm{e}_{m n}^{B C 1}-I\right) v_{3}+\left(\mathrm{e}_{m n}^{B C 2}-\mathrm{e}_{m n}^{B C 1}\right) v_{4}+\cdots \\
& +\left(\mathrm{e}_{m n}^{B C(n-4)}-\mathrm{e}_{m n}^{B C(n-5)}\right) v_{n-2} \\
& +\left(\mathrm{e}_{m n}^{B C(n-3)}-\mathrm{e}_{m n}^{B C(n-4)}\right) v_{n-1}+\left(\mathrm{e}_{m n}^{B C(n-2)}-\mathrm{e}_{m n}^{B C(n-3)}\right) v_{n} \\
& =\varphi(-2)-\varphi(-3) \text {, } \\
& \left(\mathrm{e}_{m n}^{B C 1}-I\right) v_{2}+\left(\mathrm{e}_{m n}^{B C 2}-\mathrm{e}_{m n}^{B C 1}\right) v_{3}+\cdots \\
& +\left(\mathrm{e}_{m n}^{B C(n-3)}-\mathrm{e}_{m n}^{B C(n-4)}\right) v_{n-2} \\
& +\left(\mathrm{e}_{m n}^{B C(n-2)}-\mathrm{e}_{m n}^{B C(n-3)}\right) v_{n-1}+\left(\mathrm{e}_{m n}^{B C(n-1)}-\mathrm{e}_{m n}^{B C(n-2)}\right) v_{n} \\
& =\varphi(-1)-\varphi(-2) \text {, } \\
& \left(E_{1}-E_{2}\right) \\
& \begin{aligned}
\left(\mathrm{e}_{m n}^{B C 1}-I\right) v_{1}+\left(\mathrm{e}_{m n}^{B C 2}-\mathrm{e}_{m n}^{B C 1}\right) v_{2}+\cdots \\
\quad+\left(\mathrm{e}_{m n}^{B C(n-2)}-\mathrm{e}_{m n}^{B C(n-3)}\right) v_{n-2} \\
\quad+\left(\mathrm{e}_{m n}^{B C(n-1)}-\mathrm{e}_{m n}^{B C(n-2)}\right) v_{n-1}+\left(\mathrm{e}_{m n}^{B C n}-\mathrm{e}_{m n}^{B C(n-1)}\right) v_{n} \\
=\varphi(0)-\varphi(-1)
\end{aligned} \\
& \left(E_{2}-E_{3}\right)
\end{aligned}
$$$$
\left(E_{0}-E_{1}\right)
$$

By Definition 3, we have

$$
\mathrm{e}_{m n}^{B C 1}-I=I+B+C-I=B+C,
$$

and, from the foregoing equations, we get

$$
\begin{gathered}
v_{n}=(B+C)^{-1} \Delta \varphi(-n), \\
v_{n-1}=(B+C)^{-1}\left[\Delta \varphi(-n+1)-\left(\mathrm{e}_{m n}^{B C 2}-\mathrm{e}_{m n}^{B C 1}\right) v_{n}\right], \\
v_{n-2}=(B+C)^{-1}\left[\Delta \varphi(-n+2)-\left(\mathrm{e}_{m n}^{B C 2}-\mathrm{e}_{m n}^{B C 1}\right) v_{n-1}\right. \\
\left.-\left(\mathrm{e}_{m n}^{B C 3}-\mathrm{e}_{m n}^{B C 2}\right) v_{n}\right],
\end{gathered}
$$$$
v_{3}=(B+C)^{-1}\left[\Delta \varphi(-3)-\left(\mathrm{e}_{m n}^{B C 2}-\mathrm{e}_{m n}^{B C 1}\right) v_{4}-\cdots\right.
$$$$
-\left(\mathrm{e}_{m n}^{B C(n-4)}-\mathrm{e}_{m n}^{B C(n-5)}\right) v_{n-2}
$$$$
-\left(\mathrm{e}_{m n}^{B C(n-3)}-\mathrm{e}_{m n}^{B C(n-4)}\right) v_{n-1}
$$$$
\left.-\left(\mathrm{e}_{m n}^{B C(n-2)}-\mathrm{e}_{m n}^{B C(n-3)}\right) v_{n}\right],
$$$$
v_{2}=(B+C)^{-1}\left[\Delta \varphi(-2)-\left(\mathrm{e}_{m n}^{B C 2}-\mathrm{e}_{m n}^{B C 1}\right) v_{3}-\cdots\right.
$$$$
-\left(\mathrm{e}_{m n}^{B C(n-3)}-\mathrm{e}_{m n}^{B C(n-4)}\right) v_{n-2}
$$$$
-\left(\mathrm{e}_{m n}^{B C(n-2)}-\mathrm{e}_{m n}^{B C(n-3)}\right) v_{n-1}
$$$$
\left.-\left(\mathrm{e}_{m n}^{B C(n-1)}-\mathrm{e}_{m n}^{B C(n-2)}\right) v_{n}\right],
$$$$
v_{1}=(B+C)^{-1}\left[\Delta \varphi(-1)-\left(\mathrm{e}_{m n}^{B C 2}-\mathrm{e}_{m n}^{B C 1}\right) v_{2}-\cdots\right.
$$$$
-\left(\mathrm{e}_{m n}^{B C(n-2)}-\mathrm{e}_{m n}^{B C(n-3)}\right) v_{n-2}
$$$$
-\left(\mathrm{e}_{m n}^{B C(n-1)}-\mathrm{e}_{m n}^{B C(n-2)}\right) v_{n-1}
$$$$
\left.-\left(\mathrm{e}_{m n}^{B C n}-\mathrm{e}_{m n}^{B C(n-1)}\right) v_{n}\right] \text {. }
$$

The previous formulas can be shortened as

$$
\begin{aligned}
v_{\ell} & =(B+C)^{-1}\left[\Delta \varphi(-\ell)-\sum_{t=1}^{n-\ell}\left(\mathrm{e}_{m n}^{B C(t+1)}-\mathrm{e}_{m n}^{B C t}\right) v_{t+\ell}\right] \\
& =(B+C)^{-1}\left[\Delta \varphi(-\ell)-\sum_{t=1}^{n-\ell} \Delta \mathrm{e}_{m n}^{B C t} v_{t+\ell}\right]
\end{aligned}
$$

where $\ell \in \mathbb{Z}_{1}^{n}$. Finally, from $\left(E_{n}\right)$, we get

$$
v_{0}=\varphi(-n)-\sum_{s=1}^{n} v_{s} .
$$

Theorem 10 is proved.

Now we express the solution of the homogeneous Cauchy problem by $\widetilde{\mathrm{e}}_{m n}^{B C(k)}$. In this case, the condition $\operatorname{det}(B+C) \neq 0$ is not necessary. 
Theorem 11. Let $B, C$ be constant $r \times r$ matrices with $B C=C B$ and let $m, n \in \mathbb{N}, m<n$, be fixed integers. Then the solution of the initial Cauchy problem (70), (71) can be expressed in the form

$$
x(k)=\sum_{j=0}^{n} \widetilde{\mathrm{e}}_{m n}^{B C(k+j)} w_{j},
$$

where $k \in \mathbb{Z}_{-n}^{\infty}$ and

$$
\begin{aligned}
w_{\ell}= & \Delta \varphi(-\ell-1)-\Delta \widetilde{\mathrm{e}}_{m n}^{B C(-\ell+n-1)} \varphi(-n) \\
& \quad-\sum_{s=-n}^{-\ell-m-2} \Delta \widetilde{\mathrm{e}}_{m n}^{B C(-\ell-s-2)} \Delta \varphi(s), \quad \ell \in \mathbb{Z}_{0}^{n-m-1}, \\
w_{\ell}= & \Delta \varphi(-\ell-1), \quad \ell \in \mathbb{Z}_{n-m}^{n-1}, \\
w_{n}= & \varphi(-n) .
\end{aligned}
$$

Proof. We are going to find the solution of the problem (70), (71) in the form

$$
x(k)=\sum_{j=0}^{n} \widetilde{\mathrm{e}}_{m n}^{B C(k+j)} w_{j}, \quad k \geq 0
$$

with unknown constant vectors $w_{j}$. Due to linearity (taking into account that $k$ varies), we have

$$
\begin{aligned}
\Delta x(k) & =\Delta \sum_{j=0}^{n} \widetilde{\mathrm{e}}_{m n}^{B C(k+j)} w_{j}=\sum_{j=0}^{n} \Delta\left[\widetilde{\mathrm{e}}_{m n}^{B C(k+j)} w_{j}\right] \\
& =\sum_{j=0}^{n} \Delta\left[\widetilde{\mathrm{e}}_{m n}^{B C(k+j)}\right] w_{j} .
\end{aligned}
$$

We use formula (15) and we get

$$
\begin{aligned}
\Delta x(k) & =\sum_{j=0}^{n}\left(B \widetilde{\mathrm{e}}_{m n}^{B C(k-m+j)}+C \widetilde{\mathrm{e}}_{m n}^{B C(k-n+j)}\right) w_{j} \\
& =B \sum_{j=0}^{n} \widetilde{\mathrm{e}}_{m n}^{B C(k-m+j)} w_{j}+C \sum_{j=0}^{n} \widetilde{\mathrm{e}}_{m n}^{B C(k-n+j)} w_{j} \\
& =B x(k-m)+C x(k-n) .
\end{aligned}
$$

Now we conclude that, for any $w_{j}$ and $k \in \mathbb{Z}_{0}^{\infty}$, the equation $\Delta x(k)=B x(k-m)+C x(k-n)$ holds. We will try to satisfy initial conditions (71). Due to (83), we have, for $k \in \mathbb{Z}_{-n}^{0}$

$$
\begin{aligned}
\widetilde{\mathrm{e}}_{m n}^{B C 0} w_{0} & +\widetilde{\mathrm{e}}_{m n}^{B C 1} w_{1}+\widetilde{\mathrm{e}}_{m n}^{B C 2} w_{2}+\cdots \\
+ & \widetilde{\mathrm{e}}_{m n}^{B C(n-2)} w_{n-2}+\widetilde{\mathrm{e}}_{m n}^{B C(n-1)} w_{n-1} \\
& +\widetilde{\mathrm{e}}_{m n}^{B C n} w_{n}=\varphi(0),
\end{aligned}
$$

$$
\begin{aligned}
& \widetilde{\mathrm{e}}_{m n}^{B C(-1)} w_{0}+\widetilde{\mathrm{e}}_{m n}^{B C 0} w_{1}+\widetilde{\mathrm{e}}_{m n}^{B C 1} w_{2}+\cdots \\
& +\widetilde{\mathrm{e}}_{m n}^{B C(n-3)} w_{n-2}+\widetilde{\mathrm{e}}_{m n}^{B C(n-2)} w_{n-1} \\
& +\widetilde{\mathrm{e}}_{m n}^{B C(n-1)} w_{n}=\varphi(-1), \\
& \widetilde{\mathrm{e}}_{m n}^{B C(-2)} w_{0}+\widetilde{\mathrm{e}}_{m n}^{B C(-1)} w_{1}+\widetilde{\mathrm{e}}_{m n}^{B C 0} w_{2}+\cdots \\
& +\widetilde{\mathrm{e}}_{m n}^{B C(n-4)} w_{n-2}+\widetilde{\mathrm{e}}_{m n}^{B C(n-3)} w_{n-1} \\
& +\widetilde{\mathrm{e}}_{m n}^{B C(n-2)} w_{n}=\varphi(-2), \\
& \widetilde{\mathrm{e}}_{m n}^{B C(-3)} w_{0}+\widetilde{\mathrm{e}}_{m n}^{B C(-2)} w_{1}+\widetilde{\mathrm{e}}_{m n}^{B C(-1)} w_{2}+\cdots \\
& +\widetilde{\mathrm{e}}_{m n}^{B C(n-5)} w_{n-2}+\widetilde{\mathrm{e}}_{m n}^{B C(n-4)} w_{n-1} \\
& +\widetilde{\mathrm{e}}_{m n}^{B C(n-3)} w_{n}=\varphi(-3), \\
& \widetilde{\mathrm{e}}_{m n}^{B C(-n+3)} w_{0}+\widetilde{\mathrm{e}}_{m n}^{B C(-n+4)} w_{1}+\widetilde{\mathrm{e}}_{m n}^{B C(-n+5)} w_{2}+\cdots \\
& +\widetilde{\mathrm{e}}_{m n}^{B C 1} w_{n-2}+\widetilde{\mathrm{e}}_{m n}^{B C 2} w_{n-1} \\
& +\widetilde{\mathrm{e}}_{m n}^{B C 3} w_{n}=\varphi(-n+3), \\
& \widetilde{\mathrm{e}}_{m n}^{B C(-n+2)} w_{0}+\widetilde{\mathrm{e}}_{m n}^{B C(-n+3)} w_{1}+\widetilde{\mathrm{e}}_{m n}^{B C(-n+4)} w_{2}+\cdots \\
& +\widetilde{\mathrm{e}}_{m n}^{B C 0} w_{n-2}+\widetilde{\mathrm{e}}_{m n}^{B C 1} w_{n-1} \\
& +\widetilde{\mathrm{e}}_{m n}^{B C 2} w_{n}=\varphi(-n+2), \\
& \widetilde{\mathrm{e}}_{m n}^{B C(-n+1)} w_{0}+\widetilde{\mathrm{e}}_{m n}^{B C(-n+2)} w_{1}+\widetilde{\mathrm{e}}_{m n}^{B C(-n+3)} w_{2}+\cdots \\
& +\widetilde{\mathrm{e}}_{m n}^{B C(-1)} w_{n-2}+\widetilde{\mathrm{e}}_{m n}^{B C 0} w_{n-1} \\
& +\widetilde{\mathrm{e}}_{m n}^{B C 1} w_{n}=\varphi(-n+1), \\
& \widetilde{\mathrm{e}}_{m n}^{B C(-n)} w_{0}+\widetilde{\mathrm{e}}_{m n}^{B C(-n+1)} w_{1}+\widetilde{\mathrm{e}}_{m n}^{B C(-n+2)} w_{2}+\cdots \\
& +\widetilde{\mathrm{e}}_{m n}^{B C(-2)} w_{n-2}+\widetilde{\mathrm{e}}_{m n}^{B C(-1)} w_{n-1} \\
& +\widetilde{\mathrm{e}}_{m n}^{B C 0} w_{n}=\varphi(-n) \text {. }
\end{aligned}
$$

By Definition 6, we have $\widetilde{\mathrm{e}}_{m n}^{B C k}=\Theta$ for $k \in \mathbb{Z}_{-\infty}^{-1}$ and $\widetilde{\mathrm{e}}_{m n}^{B C k}=I$ for $k \in \mathbb{Z}_{0}^{m}$. Thus, we have

$$
\begin{aligned}
& w_{0}+ w_{1}+w_{2}+\cdots+w_{m}+\widetilde{\mathrm{e}}_{m n}^{B C(m+1)} w_{m+1} \\
&+\widetilde{\mathrm{e}}_{m n}^{B C(m+2)} w_{m+2}+\cdots+\widetilde{\mathrm{e}}_{m n}^{B C(n-2)} w_{n-2} \\
&+\widetilde{\mathrm{e}}_{m n}^{B C(n-1)} w_{n-1}+\widetilde{\mathrm{e}}_{m n}^{B C n} w_{n}=\varphi(0) \\
& w_{1}+w_{2}+w_{3}+\cdots+w_{m+1}+\widetilde{\mathrm{e}}_{m n}^{B C(m+1)} w_{m+2} \\
&+\widetilde{\mathrm{e}}_{m n}^{B C(m+2)} w_{m+3}+\cdots+\widetilde{\mathrm{e}}_{m n}^{B C(n-3)} w_{n-2} \\
&+\widetilde{\mathrm{e}}_{m n}^{B C(n-2)} w_{n-1}+\widetilde{\mathrm{e}}_{m n}^{B C(n-1)} w_{n}=\varphi(-1),
\end{aligned}
$$




$$
\begin{gathered}
w_{2}+w_{3}+w_{4}+\cdots+w_{m+2}+\widetilde{\mathrm{e}}_{m n}^{B C(m+1)} w_{m+3} \\
+\widetilde{\mathrm{e}}_{m n}^{B C(m+2)} w_{m+4}+\cdots+\widetilde{\mathrm{e}}_{m n}^{B C(n-4)} w_{n-2} \quad\left(\widetilde{E}_{2}\right) \\
+\widetilde{\mathrm{e}}_{m n}^{B C(n-3)} w_{n-1}+\widetilde{\mathrm{e}}_{m n}^{B C(n-2)} w_{n}=\varphi(-2), \\
\vdots \\
w_{n-m-2}+w_{n-m-1}+w_{n-m}+\cdots+w_{n-2} \\
+\widetilde{\mathrm{e}}_{m n}^{B C(m+1)} w_{n-1}+\widetilde{\mathrm{e}}_{m n}^{B C(m+2)} w_{n}=\varphi(-n+m+2), \\
w_{n-m-1}+w_{n-m}+w_{n-m+1}+\cdots+w_{n-2} \\
+w_{n-1}+\widetilde{\mathrm{e}}_{m n}^{B C(m+1)} w_{n}=\varphi(-n+m+1), \quad\left(\widetilde{E}_{n-m-2}\right) \\
w_{n-m}+w_{n-m+1}+w_{n-m+2}+\cdots \\
+w_{n-2}+w_{n-1}+w_{n}=\varphi(-n+m), \\
\vdots \\
w_{n-2}+w_{n-1}+w_{n}=\varphi(-n+2), \\
w_{n-1}+w_{n}=\varphi(-n+1), \\
w_{n}=\varphi(-n) . \\
\left(\widetilde{E}_{n-m}\right) \\
\left(\widetilde{E}_{n-2}\right) \\
\left(\widetilde{E}_{n-1}\right) \\
\left(\widetilde{E}_{n}\right)
\end{gathered}
$$

We see directly that $w_{n}=\varphi(-n)$. Subtracting the neighbouring equations $\left(\left(\widetilde{E}_{n-1}-\widetilde{E}_{n}\right),\left(\widetilde{E}_{n-2}-\widetilde{E}_{n-1}\right), \ldots\right.$, $\left.\left(\widetilde{E}_{n-m}-\widetilde{E}_{n-m+1}\right)\right)$, we immediately get the formulas for $w_{n-1}, w_{n-2}, \ldots, w_{n-m}$ as follows:

$$
\begin{gathered}
w_{n-1}=\varphi(-n+1)-\varphi(-n)=\Delta \varphi(-n), \quad\left(\widetilde{E}_{n-1}-\widetilde{E}_{n}\right) \\
w_{n-2}=\varphi(-n+2)-\varphi(-n+1)=\Delta \varphi\left(\begin{array}{r}
-n+1), \\
\left(\widetilde{E}_{n-2}-\widetilde{E}_{n-1}\right)
\end{array}\right. \\
\vdots \\
\begin{array}{rr}
w_{n-m+1}=\varphi(-n+m-1)-\varphi(-n+m-2) \\
=\Delta \varphi(-n+m-2),
\end{array} \\
w_{n-m}=\varphi(-n+m)-\varphi(-n+m-1) \\
=\Delta \varphi(-n+m-1) .
\end{gathered}
$$

Further, subtracting the neighbouring equations $\left(\left(\widetilde{E}_{n-m-1}-\widetilde{E}_{n-m}\right),\left(\widetilde{E}_{n-m-2}-\widetilde{E}_{n-m-1}\right), \ldots,\left(\widetilde{E}_{0}-\widetilde{E}_{1}\right)\right)$, we get

$$
\begin{aligned}
& w_{n-m-1}+\left[\widetilde{\mathrm{e}}_{m n}^{B C(m+1)}-I\right] w_{n} \\
& \quad=\varphi(-n+m+1)-\varphi(-n+m) \\
& \Longrightarrow w_{n-m-1}=\Delta \varphi(-n+m)-\left[\widetilde{\mathrm{e}}_{m n}^{B C(m+1)}-I\right] \varphi(-n), \\
& \quad\left(\widetilde{E}_{n-m-1}-\widetilde{E}_{n-m}\right)
\end{aligned}
$$

$$
\begin{gathered}
w_{n-m-2}+\left[\widetilde{\mathrm{e}}_{m n}^{B C(m+1)}-I\right] w_{n-1} \\
+\left[\widetilde{\mathrm{e}}_{m n}^{B C(m+2)}-\widetilde{\mathrm{e}}_{m n}^{B C(m+1)}\right] w_{n} \\
=\varphi(-n+m+2)-\varphi(-n+m+1) \\
\Longrightarrow w_{n-m-2}=\Delta \varphi(-n+m+1) \\
-\left[\widetilde{\mathrm{e}}_{m n}^{B C(m+2)}-\widetilde{\mathrm{e}}_{m n}^{B C(m+1)}\right] \varphi(-n) \\
-\left[\widetilde{\mathrm{e}}_{m n}^{B C(m+1)}-I\right] \Delta \varphi(-n),
\end{gathered}
$$$$
\left(\widetilde{E}_{n-m-2}-\widetilde{E}_{n-m-1}\right)
$$

$$
\begin{aligned}
w_{2}+ & {\left[\widetilde{\mathrm{e}}_{m n}^{B C(m+1)}-I\right] w_{m+3} } \\
& +\left[\widetilde{\mathrm{e}}_{m n}^{B C(m+2)}-\widetilde{\mathrm{e}}_{m n}^{B C(m+1)}\right] w_{m+4}+\cdots \\
& +\left[\widetilde{\mathrm{e}}_{m n}^{B C(n-4)}-\widetilde{\mathrm{e}}_{m n}^{B C(n-5)}\right] w_{n-2} \\
& +\left[\widetilde{\mathrm{e}}_{m n}^{B C(n-3)}-\widetilde{\mathrm{e}}_{m n}^{B C(n-4)}\right] w_{n-1} \\
& +\left[\widetilde{\mathrm{e}}_{m n}^{B C(n-2)}-\widetilde{\mathrm{e}}_{m n}^{B C(n-3)}\right] w_{n}=\varphi(-2)-\varphi(-3) \\
\Longrightarrow & w_{2}=\Delta \varphi(-3)-\left[\widetilde{\mathrm{e}}_{m n}^{B C(n-2)}-\widetilde{\mathrm{e}}_{m n}^{B C(n-3)}\right] \varphi(-n) \\
& -\left[\widetilde{\mathrm{e}}_{m n}^{B C(n-3)}-\widetilde{\mathrm{e}}_{m n}^{B C(n-4)}\right] \Delta \varphi(-n) \\
& -\left[\widetilde{\mathrm{e}}_{m n}^{B C(n-4)}-\widetilde{\mathrm{e}}_{m n}^{B C(n-5)}\right] \Delta \varphi(-n+1)-\cdots \\
& -\left[\widetilde{\mathrm{e}}_{m n}^{B C(m+2)}-\widetilde{\mathrm{e}}_{m n}^{B C(m+1)}\right] \Delta \varphi(-m-5) \\
& -\left[\widetilde{\mathrm{e}}_{m n}^{B C(m+1)}-I\right] \Delta \varphi(-m-4) \\
& \left(\widetilde{E}_{2}-\widetilde{E}_{3}\right)
\end{aligned}
$$$$
w_{1}+\left[\widetilde{\mathrm{e}}_{m n}^{B C(m+1)}-I\right] w_{m+2}+\left[\widetilde{\mathrm{e}}_{m n}^{B C(m+2)}-\widetilde{\mathrm{e}}_{m n}^{B C(m+1)}\right] w_{m+3}
$$$$
+\cdots+\left[\widetilde{\mathrm{e}}_{m n}^{B C(n-3)}-\widetilde{\mathrm{e}}_{m n}^{B C(n-4)}\right] w_{n-2}
$$$$
+\left[\widetilde{\mathrm{e}}_{m n}^{B C(n-2)}-\widetilde{\mathrm{e}}_{m n}^{B C(n-3)}\right] w_{n-1}
$$$$
+\left[\widetilde{\mathrm{e}}_{m n}^{B C(n-1)}-\widetilde{\mathrm{e}}_{m n}^{B C(n-2)}\right] w_{n}
$$$$
=\varphi(-1)-\varphi(-2)
$$$$
\Longrightarrow w_{1}=\Delta \varphi(-2)
$$$$
-\left[\widetilde{\mathrm{e}}_{m n}^{B C(n-1)}-\widetilde{\mathrm{e}}_{m n}^{B C(n-2)}\right] \varphi(-n)
$$ 


$$
\begin{aligned}
& -\left[\widetilde{\mathrm{e}}_{m n}^{B C(n-2)}-\widetilde{\mathrm{e}}_{m n}^{B C(n-3)}\right] \Delta \varphi(-n) \\
& -\left[\widetilde{\mathrm{e}}_{m n}^{B C(n-3)}-\widetilde{\mathrm{e}}_{m n}^{B C(n-4)}\right] \Delta \varphi(-n+1)-\cdots \\
& -\left[\widetilde{\mathrm{e}}_{m n}^{B C(m+2)}-\widetilde{\mathrm{e}}_{m n}^{B C(m+1)}\right] \Delta \varphi(-m-4) \\
& -\left[\widetilde{\mathrm{e}}_{m n}^{B C(m+1)}-I\right] \Delta \varphi(-m-3), \\
w_{0}+ & {\left[\widetilde{\mathrm{e}}_{m n}^{B C(m+1)}-I\right] w_{m+1} } \\
& +\left[\widetilde{\mathrm{e}}_{m n}^{B C(m+2)}-\widetilde{\mathrm{e}}_{m n}^{B C(m+1)}\right] w_{m+2}+\cdots \\
& +\left[\widetilde{\mathrm{e}}_{m n}^{B C(n-2)}-\widetilde{\mathrm{e}}_{m n}^{B C(n-3)}\right] w_{n-2} \\
& +\left[\widetilde{\mathrm{e}}_{m n}^{B C(n-1)}-\widetilde{\mathrm{e}}_{m n}^{B C(n-2)}\right] w_{n-1} \\
& +\left[\widetilde{\mathrm{e}}_{m n}^{B C n}-\widetilde{\mathrm{e}}_{m n}^{B C(n-1)}\right] w_{n} \\
= & \varphi(0)-\varphi(-1) \\
\Longrightarrow & w_{0}=\Delta \varphi(-1)-\left[\widetilde{\mathrm{e}}_{m n}^{B C n}-\widetilde{\mathrm{e}}_{m n}^{B C(n-1)}\right] \varphi(-n) \\
& -\left[\widetilde{\mathrm{e}}_{m n}^{B C(n-1)}-\widetilde{\mathrm{e}}_{m n}^{B C(n-2)}\right] \Delta \varphi(-n) \\
& -\left[\widetilde{\mathrm{e}}_{m n}^{B C(n-2)}-\widetilde{\mathrm{e}}_{m n}^{B C(n-3)}\right] \Delta \varphi(-n+1)-\cdots \\
& -\left[\widetilde{\mathrm{e}}_{m n}^{B C(m+2)}-\widetilde{\mathrm{e}}_{m n}^{B C(m+1)}\right] \Delta \varphi(-m-3) \\
& -\left[\widetilde{\mathrm{e}}_{m n}^{B C(m+1)}-I\right] \Delta \varphi(-m-2) .
\end{aligned}
$$

The previous formulas can be written as

$$
\begin{aligned}
w_{\ell}= & \Delta \varphi(-\ell-1)-\left[\widetilde{\mathrm{e}}_{m n}^{B C(-\ell+n)}-\widetilde{\mathrm{e}}_{m n}^{B C(-\ell+n-1)}\right] \varphi(-n) \\
& -\sum_{s=-n}^{-\ell-m-2}\left[\widetilde{\mathrm{e}}_{m n}^{B C(-\ell-s-1)}-\widetilde{\mathrm{e}}_{m n}^{B C(-\ell-s-2)}\right] \Delta \varphi(s), \\
= & \Delta \varphi(-\ell-1)-\Delta \widetilde{\mathrm{e}}_{m n}^{B C(-\ell+n-1)} \varphi(-n) \\
& -\sum_{s=-n}^{-\ell-m-2} \Delta \widetilde{\mathrm{e}}_{m n}^{B C(-\ell-s-2)} \Delta \varphi(s), \quad \ell \in \mathbb{Z}_{0}^{n-m-1}, \\
w_{\ell}= & \Delta \varphi(-\ell-1), \quad \ell \in \mathbb{Z}_{n-m}^{n-1}, \\
w_{n}= & \varphi(-n) .
\end{aligned}
$$

Theorem 11 is proved.

3.2. Representing the Solution of a Nonhomogeneous Initial Problem. We consider a nonhomogeneous initial Cauchy problem

$$
\begin{gathered}
\Delta x(k)=B x(k-m)+C x(k-n)+f(k), \quad k \in \mathbb{Z}_{0}^{\infty}, \\
x(k)=\varphi(k), \quad k \in \mathbb{Z}_{-n}^{0} .
\end{gathered}
$$

By the theory of linear equations, we can obtain its solution as the sum of a solution of adjoint homogeneous problem (70), (71) (satisfying the same initial data) and a particular solution of (90) being zero on an initial interval. Let us, therefore, find such a particular solution.

We need an auxiliary lemma the proof of which is omitted.

Lemma 12. Let a function $F(k, n)$ of two discrete variables be given. Then,

$$
\Delta_{k}\left[\sum_{j=1}^{k} F(k, j)\right]=F(k+1, k+1)+\sum_{j=1}^{k} \Delta_{k} F(k, j) .
$$

Now we are ready to find a particular solution $x_{p}(k), k \in$ $\mathbb{Z}_{-n}^{\infty}$ of the initial Cauchy problem:

$$
\begin{gathered}
\Delta x(k)=B x(\mathrm{k}-m)+C x(k-n)+f(k), \quad k \in \mathbb{Z}_{0}^{\infty}, \\
x(k)=0, \quad k \in \mathbb{Z}_{-n}^{0} .
\end{gathered}
$$

Theorem 13. The solution $x=x_{p}(k)$ of the initial Cauchy problem (93), (94) can be represented on $\mathbb{Z}_{-n}^{\infty}$ in the form

$$
x_{p}(k)=\sum_{\ell=1}^{k} \widetilde{\mathrm{e}}_{m n}^{B C(k-\ell)} f(\ell-1), k \in \mathbb{Z}_{0}^{\infty} .
$$

Proof. We are going to find a particular solution $x_{p}(k)$ of problem (93), (94) in the form (95). We substitute (95) into (93). Then, we get

$$
\begin{aligned}
\Delta\left[\sum_{\ell=1}^{k} \widetilde{\mathrm{e}}_{m n}^{B C(k-\ell)} f(\ell-1)\right] \\
=B \sum_{\ell=1}^{k-m} \widetilde{\mathrm{e}}_{m n}^{B C(k-m-\ell)} f(\ell-1) \\
\quad+C \sum_{\ell=1}^{k-n} \widetilde{\mathrm{e}}_{m n}^{B C(k-n-\ell)} f(j-1)+f(k) .
\end{aligned}
$$

We modify the left-hand side of (96). With the aid of Lemma 12, we obtain

$$
\begin{aligned}
\Delta\left[\sum_{\ell=1}^{k} \widetilde{\mathrm{e}}_{m n}^{B C(k-\ell)} f(j-1)\right] \\
=\widetilde{\mathrm{e}}_{m n}^{B C((k+1)-(k+1))} f(k+1-1) \\
\quad+\sum_{\ell=1}^{k} \Delta\left[\widetilde{\mathrm{e}}_{m n}^{B C(k-\ell)} f(j-1)\right],
\end{aligned}
$$


and, applying Theorem 9, we get

$$
\begin{aligned}
& \Delta\left[\sum_{\ell=1}^{k} \widetilde{\mathrm{e}}_{m n}^{B C(k-\ell)} f(j-1)\right] \\
& =\widetilde{\mathrm{e}}_{m n}^{B C 0} f(k)+\sum_{\ell=1}^{k}\left[B \widetilde{\mathrm{e}}_{m n}^{B C(k-m-\ell)}+C \widetilde{\mathrm{e}}_{m n}^{B C(k-n-\ell)}\right] f(j-1) \\
& =\widetilde{\mathrm{e}}_{m n}^{B C 0} f(k)+B\left[\sum_{\ell=1}^{k-m} \widetilde{\mathrm{e}}_{m n}^{B C(k-m-\ell)} f(j-1)\right. \\
& +C\left[\sum_{\ell=1}^{k-n} \widetilde{\mathrm{e}}_{m n}^{B C(k-n-\ell)} f(j-1)\right. \\
& \left.+\sum_{\ell=k-n+1}^{k} \widetilde{\mathrm{e}}_{m n}^{B C(k-m-\ell)} f(j-1)\right]
\end{aligned}
$$

By Definition 6, we have $\widetilde{\mathrm{e}}_{m n}^{B C 0}=I, \widetilde{\mathrm{e}}_{m n}^{B C(k-m-\ell)}=\Theta$ for $\ell \epsilon$ $\mathbb{Z}_{k-m+1}^{k}$ and $\widetilde{\mathrm{e}}_{m n}^{B C(k-n-\ell)}=\Theta$ for $\ell \in \mathbb{Z}_{k-n+1}^{k}$. Thus, we get

$$
\begin{aligned}
& \Delta\left[\sum_{\ell=1}^{k} \widetilde{\mathrm{e}}_{m n}^{B C(k-\ell)} f(j-1)\right] \\
& =f(k)+B \sum_{\ell=1}^{k-m} \widetilde{\mathrm{e}}_{m n}^{B C(k-m-\ell)} f(j-1) \\
& +C \sum_{\ell=1}^{k-n} \widetilde{\mathrm{e}}_{m n}^{B C(k-n-\ell)} f(j-1)
\end{aligned}
$$

and (96) holds.

Combining the results of Theorems 10, 11, and 13, we get immediately the following two theorems, which describe the solution of (90), (91). The first theorem uses the delayed matrix exponential $\mathrm{e}_{m n}^{B C k}$ and the second one uses the delayed matrix exponential $\widetilde{\mathrm{e}}_{m n}^{B C k}$.

Theorem 14. Let $B, C$ be constant $r \times r$ matrices with

$$
B C=C B, \quad \operatorname{det}(B+C) \neq 0,
$$

and let $m, n \in \mathbb{N}, m<n$, be fixed integers. Then, the solution of the initial Cauchy problem (90), (91) can be expressed in the form

$$
x(k)=\sum_{j=0}^{n} e_{m n}^{B C(k+j)} v_{j}+\sum_{\ell=1}^{k} \widetilde{\mathrm{e}}_{m n}^{B C(k-\ell)} f(\ell-1),
$$

where $k \in \mathbb{Z}_{-n}^{\infty}$ and

$$
\begin{gathered}
v_{0}=\varphi(-n)-\sum_{s=1}^{n} v_{s} \\
v_{\ell}=(B+C)^{-1}\left[\Delta \varphi(-\ell)-\sum_{t=1}^{n-\ell} \Delta e_{m n}^{B C t} v_{t+\ell}\right], \quad \ell \in \mathbb{Z}_{1}^{n} .
\end{gathered}
$$

Theorem 15. Let $B, C$ be constant $r \times r$ matrices with $B C=C B$ and let $m, n \in \mathbb{N}, m<n$, be fixed integers. Then, the solution of the initial Cauchy problem (90), (91) can be expressed in the form

$$
x(k)=\sum_{j=0}^{n} \widetilde{e}_{m n}^{B C(k+j)} w_{j}+\sum_{\ell=1}^{k} \widetilde{e}_{m n}^{B C(k-\ell)} f(\ell-1),
$$

where $k \in \mathbb{Z}_{-n}^{\infty}$ and

$$
\begin{aligned}
w_{\ell}= & \Delta \varphi(-\ell-1)-\Delta \widetilde{e}_{m n}^{B C(-\ell+n-1)} \varphi(-n) \\
& -\sum_{s=-n}^{-\ell-m-2} \Delta \widetilde{e}_{m n}^{B C(-\ell-s-2)} \Delta \varphi(s), \quad \ell \in \mathbb{Z}_{0}^{n-m-1}, \\
w_{\ell} & =\Delta \varphi(-\ell-1), \quad \ell \in \mathbb{Z}_{n-m}^{n-1}, \\
w_{n}= & \varphi(-n) .
\end{aligned}
$$

\section{Examples}

Below, we show four examples to demonstrate the results achieved.

Example 16. Let us represent the solution of the scalar $(r=1)$ problem (70), (71) where we put $m=2, n=3, B=b, C=$ $c, \varphi(-3)=1, \varphi(-2)=2, \varphi(-1)=3$, and $\varphi(0)=4$, using Theorem 10. We get

$$
\begin{gathered}
\Delta x(k)=b x(k-2)+c x(k-3), \quad k \in \mathbb{Z}_{0}^{\infty}, \\
x(-3)=\varphi(-3)=1, \\
x(-2)=\varphi(-2)=2, \\
x(-1)=\varphi(-1)=3, \\
x(0)=\varphi(0)=4 .
\end{gathered}
$$

By Theorem 10, the solution of problem (105), (106) is

$$
x(k)=\sum_{j=0}^{3} \mathrm{e}_{2,3}^{b c(k+j)} v_{j}, \quad k \in \mathbb{Z}_{-3}^{\infty},
$$


where

$$
\begin{aligned}
v_{3}=(b+c)^{-1}\left[\Delta \varphi(-3)-\sum_{t=1}^{0} \Delta \mathrm{e}_{2,3}^{b c t} v_{t+3}\right]=(b+c)^{-1} \\
v_{2}=(b+c)^{-1}\left[\Delta \varphi(-2)-\sum_{t=1}^{1} \Delta \mathrm{e}_{2,3}^{b c t} v_{t+2}\right] \\
=(b+c)^{-1}\left[\Delta \varphi(-2)-\Delta \mathrm{e}_{2,3}^{b c 1} v_{3}\right] \\
=(b+c)^{-1}\left[1-\left(\mathrm{e}_{2,3}^{b c 2}-\mathrm{e}_{2,3}^{b c 1}\right)(b+c)^{-1}\right] \\
=(b+c)^{-1}\left[1-(b+c)(b+c)^{-1}\right]=0, \\
v_{1}=(b+c)^{-1}\left[\Delta \varphi(-1)-\sum_{t=1}^{2} \Delta \mathrm{e}_{2,3}^{b c t} v_{t+1}\right] \\
=(b+c)^{-1}\left[\Delta \varphi(-2)-\Delta \mathrm{e}_{2,3}^{b c 1} v_{2}-\Delta \mathrm{e}_{2,3}^{b c 2} v_{3}\right] \\
=(b+c)^{-1}\left[1-\left(\mathrm{e}_{2,3}^{b c 3}-\mathrm{e}_{2,3}^{b c 2}\right)(b+c)^{-1}\right] \\
=(b+c)^{-1}\left[1-(b+c)(b+c)^{-1}\right]=0, \\
v_{0}=\varphi(-3)-\sum_{s=1}^{3} v_{s}=1-(b+c)^{-1} .
\end{aligned}
$$

Thus, we get

$$
x(k)=\mathrm{e}_{2,3}^{b c k}\left[1-(b+c)^{-1}\right]+\mathrm{e}_{2,3}^{b c(k+3)}(b+c)^{-1} .
$$

We give values of $x(k)$ for $k \in \mathbb{Z}_{1}^{8}$ as follows:

$$
\begin{aligned}
& x(1)=4+2 b+c, \\
& x(2)=4+5 b+3 c \\
& x(3)=4+9 b+6 c \\
& x(4)=4+13 b+10 c+2 b^{2}+b c \\
& x(5)=4+17 b+14 c+7 b^{2}+6 b c+c^{2}, \\
& x(6)=4+21 b+18 c+16 b^{2}+17 b c+4 c^{2}, \\
& x(7)=4+25 b+22 c+29 b^{2}+36 b c+10 c^{2}+2 b^{3}+b^{2} c, \\
& x(8)=4+29 b+26 c+46 b^{2}+63 b c+20 c^{2} \\
& \quad+9 b^{3}+9 b^{2} c+2 b c^{2} .
\end{aligned}
$$

Example 17. Let us represent the solution of the scalar $(r=1)$ problem (90), (91) where we put $m=2, n=3, B=b, C=c$, $\varphi(-3)=1, \varphi(-2)=2, \varphi(-1)=3, \varphi(0)=4$, and $f(k)=k+1$, using Theorem 11. Thus, we have

$$
\begin{gathered}
\Delta x(k)=b x(k-2)+c x(k-3)+k+1, \quad k \in \mathbb{Z}_{0}^{\infty}, \\
x(-3)=\varphi(-3)=1, \\
x(-2)=\varphi(-2)=2, \\
x(-1)=\varphi(-1)=3, \\
x(0)=\varphi(0)=4 .
\end{gathered}
$$

By Theorem 11, the solution of problem (111), (112) is

$$
x(k)=\sum_{j=0}^{3} \widetilde{\mathrm{e}}_{2,3}^{b c(k+j)} w_{j}+\sum_{\ell=1}^{k} \widetilde{\mathrm{e}}_{2,3}^{b c(k-\ell)} \ell, \quad k \in \mathbb{Z}_{-3}^{\infty},
$$

where

$$
\begin{gathered}
w_{0}=\Delta \varphi(-1)-\Delta \widetilde{\mathrm{e}}_{2,3}^{b c 2} \varphi(-3)-\sum_{s=-3}^{-4} \Delta \widetilde{\mathrm{e}}_{2,3}^{b c(-s-2)} \Delta \varphi(s) \\
=1-\left(\widetilde{\mathrm{e}}_{2,3}^{b c 3}-\widetilde{\mathrm{e}}_{2,3}^{b c 2}\right) 1 \\
=1-(1+b-1)=1-b, \\
w_{1}=\Delta \varphi(-2)=1, \\
w_{2}=\Delta \varphi(-3)=1, \\
w_{3}=\varphi(-3)=1 .
\end{gathered}
$$

Thus, we get

$$
\begin{gathered}
x(k)=\widetilde{\mathrm{e}}_{2,3}^{b c k}(1-b)+\widetilde{\mathrm{e}}_{2,3}^{b c(k+1)}+\widetilde{\mathrm{e}}_{2,3}^{b c(k+2)} \\
+\widetilde{\mathrm{e}}_{2,3}^{b c(k+3)}+\sum_{\ell=1}^{k} \widetilde{\mathrm{e}}_{2,3}^{b c(k-\ell)} \ell .
\end{gathered}
$$

The first eight values of the homogeneous problem are given in Example 16. Now, we compute the first eight values of a particular solution $x_{p}(k)=\sum_{\ell=1}^{k} \widetilde{\mathrm{e}}_{2,3}^{b c(k-\ell)} \ell$ as follows:

$$
\begin{gathered}
x_{p}(1)=1, \quad x_{p}(2)=3, \\
x_{p}(3)=6, \quad x_{p}(4)=10+b, \\
x_{p}(5)=15+4 b+c, \quad x_{p}(6)=21+10 b+4 c, \\
x_{p}(7)=28+20 b+10 c+b^{2}, \\
x_{p}(8)=36+35 b+20 c+5 b^{2}+2 b c .
\end{gathered}
$$


Together, we get

$$
\begin{aligned}
& x(1)=5+2 b+c, \quad x(2)=7+5 b+3 c, \\
& x(3)=10+9 b+6 c, \\
& x(4)=14+14 b+10 c+2 b^{2}+b c, \\
& x(5)=19+21 b+15 c+7 b^{2}+6 b c+c^{2}, \\
& x(6)=25+31 b+22 c+16 b^{2}+17 b c+4 c^{2}, \\
& x(7)=32+45 b+32 c+30 b^{2}+36 b c+10 c^{2}+2 b^{3}+b^{2} c, \\
& x(8)=40+64 b+46 c+51 b^{2}+65 b c+20 c^{2} \\
& +9 b^{3}+9 b^{2} c+2 b c^{2} .
\end{aligned}
$$

Example 18. Let us represent the solution of the scalar $(r=1)$ problem (70), (71) where we put $m=2, n=3, B=b=4$, $C=c=-1, \varphi(-3)=1, \varphi(-2)=2, \varphi(-1)=3$, and $\varphi(0)=4$, using Theorem 10. Thus, we have

$$
\begin{gathered}
\Delta x(k)=4 x(k-2)-x(k-3), \quad k \in \mathbb{Z}_{0}^{\infty}, \\
x(-3)=\varphi(-3)=1, \\
x(-2)=\varphi(-2)=2, \\
x(-1)=\varphi(-1)=3, \\
x(0)=\varphi(0)=4 .
\end{gathered}
$$

By Theorem 10, the solution of problem (118), (119) is

$$
x(k)=\sum_{j=0}^{3} \mathrm{e}_{2,3}^{b c(k+j)} v_{j}, \quad k \in \mathbb{Z}_{-3}^{\infty},
$$

where

$$
\begin{aligned}
v_{3}=(b+c)^{-1}\left[\Delta \varphi(-3)-\sum_{t=1}^{0} \Delta \mathrm{e}_{2,3}^{b c t} v_{t+3}\right]=(b+c)^{-1}=\frac{1}{3} \\
v_{2}=(b+c)^{-1}\left[\Delta \varphi(-2)-\sum_{t=1}^{1} \Delta \mathrm{e}_{2,3}^{b c t} v_{t+2}\right] \\
=(b+c)^{-1}\left[\Delta \varphi(-2)-\Delta \mathrm{e}_{2,3}^{b c 1} v_{3}\right] \\
=(b+c)^{-1}\left[1-\left(\mathrm{e}_{2,3}^{b c 2}-\mathrm{e}_{2,3}^{b c 1}\right)(b+c)^{-1}\right] \\
=(b+c)^{-1}\left[1-(b+c)(b+c)^{-1}\right]=0
\end{aligned}
$$

$$
\begin{aligned}
v_{1} & =(b+c)^{-1}\left[\Delta \varphi(-1)-\sum_{t=1}^{2} \Delta \mathrm{e}_{2,3}^{b c t} v_{t+1}\right] \\
& =(b+c)^{-1}\left[\Delta \varphi(-2)-\Delta \mathrm{e}_{2,3}^{b c 1} v_{2}-\Delta \mathrm{e}_{2,3}^{b c 2} v_{3}\right] \\
& =(b+c)^{-1}\left[1-\left(\mathrm{e}_{2,3}^{b c 3}-\mathrm{e}_{2,3}^{b c 2}\right)(b+c)^{-1}\right] \\
& =(b+c)^{-1}\left[1-(b+c)(b+c)^{-1}\right]=0 \\
& v_{0}=\varphi(-3)-\sum_{s=1}^{3} v_{s}=1-(b+c)^{-1}=\frac{2}{3}
\end{aligned}
$$

Thus, we get

$$
\begin{gathered}
x(k)=\mathrm{e}_{2,3}^{b c k} \cdot \frac{2}{3}+\mathrm{e}_{2,3}^{b c(k+3)} \cdot \frac{1}{3}, \\
x(1)=\mathrm{e}_{2,3}^{b c 1} \cdot \frac{2}{3}+\mathrm{e}_{2,3}^{b c 4} \cdot \frac{1}{3}=4 \cdot \frac{2}{3}+25 \cdot \frac{1}{3}=11, \\
x(2)=\mathrm{e}_{2,3}^{b c 2} \cdot \frac{2}{3}+\mathrm{e}_{2,3}^{b c 5} \cdot \frac{1}{3}=7 \cdot \frac{2}{3}+49 \cdot \frac{1}{3}=21, \\
x(3)=\mathrm{e}_{2,3}^{b c 3} \cdot \frac{2}{3}+\mathrm{e}_{2,3}^{b c 6} \cdot \frac{1}{3}=10 \cdot \frac{2}{3}+82 \cdot \frac{1}{3}=34, \\
x(4)=\mathrm{e}_{2,3}^{b c 4} \cdot \frac{2}{3}+\mathrm{e}_{2,3}^{b c 7} \cdot \frac{1}{3}=25 \cdot \frac{2}{3}+172 \cdot \frac{1}{3}=74, \\
x(5)=\mathrm{e}_{2,3}^{b c 5} \cdot \frac{2}{3}+\mathrm{e}_{2,3}^{b c 8} \cdot \frac{1}{3}=49 \cdot \frac{2}{3}+343 \cdot \frac{1}{3}=147, \\
x(6)=\mathrm{e}_{2,3}^{b c 6} \cdot \frac{2}{3}+\mathrm{e}_{2,3}^{b c 9} \cdot \frac{1}{3}=82 \cdot \frac{2}{3}+622 \cdot \frac{1}{3}=262, \\
x(7)=\mathrm{e}_{2,3}^{b c 7} \cdot \frac{2}{3}+\mathrm{e}_{2,3}^{b c 10} \cdot \frac{1}{3}=172 \cdot \frac{2}{3}+1228 \cdot \frac{1}{3}=524, \\
x(8)=\mathrm{e}_{2,3}^{b c 8} \cdot \frac{2}{3}+\mathrm{e}_{2,3}^{b c 11} \cdot \frac{1}{3}=343 \cdot \frac{2}{3}+2428 \cdot \frac{1}{3}=1038 .
\end{gathered}
$$

Example 19. Let us represent the solution of the scalar $(r=1)$ problem (90), (91) where we put $m=2, n=3, B=b=4$, $C=c=-1, \varphi(-3)=1, \varphi(-2)=2, \varphi(-1)=3, \varphi(0)=4$, and $f(k)=k+1$, using Theorem 11. Thus, we have

$$
\begin{gathered}
\Delta x(k)=4 x(k-2)-x(k-3)+k+1, \quad k \in \mathbb{Z}_{0}^{\infty}, \\
x(-3)=\varphi(-3)=1, \\
x(-2)=\varphi(-2)=2, \\
x(-1)=\varphi(-1)=3, \\
x(0)=\varphi(0)=4 .
\end{gathered}
$$

By Theorem 11, the solution of the problem (123), (124) is

$$
x(k)=\sum_{j=0}^{3} \widetilde{\mathrm{e}}_{2,3}^{b c(k+j)} w_{j}+\sum_{\ell=1}^{k} \widetilde{\mathrm{e}}_{2,3}^{b c(k-\ell)} \ell, \quad k \in \mathbb{Z}_{-3}^{\infty},
$$


where

$$
\begin{gathered}
w_{0}=\Delta \varphi(-1)-\Delta \widetilde{\mathrm{e}}_{2,3}^{b c 2} \varphi(-3)-\sum_{s=-3}^{-4} \Delta \widetilde{\mathrm{e}}_{2,3}^{b c(-s-2)} \Delta \varphi(s) \\
=1-\left(\widetilde{\mathrm{e}}_{2,3}^{b c 3}-\widetilde{\mathrm{e}}_{2,3}^{b c 2}\right) 1 \\
=1-(1+b-1)=1-b=-3 \\
w_{1}=\Delta \varphi(-2)=1 \\
w_{2}=\Delta \varphi(-3)=1 \\
w_{3}=\varphi(-3)=1
\end{gathered}
$$

Thus, we get

$$
\begin{aligned}
& x(k)=\widetilde{\mathrm{e}}_{2,3}^{b c k}(-3)+\widetilde{\mathrm{e}}_{2,3}^{b c(k+1)}+\widetilde{\mathrm{e}}_{2,3}^{b c(k+2)} \\
& +\widetilde{\mathrm{e}}_{2,3}^{b c(k+3)}+\sum_{\ell=1}^{k} \widetilde{\mathrm{e}}_{2,3}^{b c(k-\ell)} \ell, \\
& x(1)=\widetilde{\mathrm{e}}_{2,3}^{b c 1}(-3)+\widetilde{\mathrm{e}}_{2,3}^{b c 2}+\widetilde{\mathrm{e}}_{2,3}^{b c 3}+\widetilde{\mathrm{e}}_{2,3}^{b c 4}+\sum_{\ell=1}^{1} \widetilde{\mathrm{e}}_{2,3}^{b c(1-\ell)} \ell \\
& =-3+1+5+8+1=12 \text {, } \\
& x(2)=\widetilde{\mathrm{e}}_{2,3}^{b c 2}(-3)+\widetilde{\mathrm{e}}_{2,3}^{b c 3}+\widetilde{\mathrm{e}}_{2,3}^{b c 4}+\widetilde{\mathrm{e}}_{2,3}^{b c 5}+\sum_{\ell=1}^{2} \widetilde{\mathrm{e}}_{2,3}^{b c(2-\ell)} \ell \\
& =-3+5+8+11+3=24 \text {, } \\
& x(3)=\widetilde{\mathrm{e}}_{2,3}^{b c 3}(-3)+\widetilde{\mathrm{e}}_{2,3}^{b c 4}+\widetilde{\mathrm{e}}_{2,3}^{b c 5}+\widetilde{\mathrm{e}}_{2,3}^{b c 6}+\sum_{\ell=1}^{3} \widetilde{\mathrm{e}}_{2,3}^{b c(3-\ell)} \ell \\
& =-15+8+11+30+6=40 \text {, } \\
& x(4)=\widetilde{\mathrm{e}}_{2,3}^{b c 4}(-3)+\widetilde{\mathrm{e}}_{2,3}^{b c 5}+\widetilde{\mathrm{e}}_{2,3}^{b c 6}+\widetilde{\mathrm{e}}_{2,3}^{b c 7}+\sum_{\ell=1}^{4} \widetilde{\mathrm{e}}_{2,3}^{b c(4-\ell)} \ell \\
& =-24+11+30+57+14=88 \text {, } \\
& x(5)=\widetilde{\mathrm{e}}_{2,3}^{b c 5}(-3)+\widetilde{\mathrm{e}}_{2,3}^{b c 6}+\widetilde{\mathrm{e}}_{2,3}^{b c 7}+\widetilde{\mathrm{e}}_{2,3}^{b c 8}+\sum_{\ell=1}^{5} \widetilde{\mathrm{e}}_{2,3}^{b c(5-\ell)} \ell \\
& =-33+30+57+93+30=177 \text {, } \\
& x(6)=\widetilde{\mathrm{e}}_{2,3}^{b c 6}(-3)+\widetilde{\mathrm{e}}_{2,3}^{b c 7}+\widetilde{\mathrm{e}}_{2,3}^{b c 8}+\widetilde{\mathrm{e}}_{2,3}^{b c 9}+\sum_{\ell=1}^{6} \widetilde{\mathrm{e}}_{2,3}^{b c(6-\ell)} \ell \\
& =-90+57+93+202+57=319 \text {, } \\
& x(7)=\widetilde{\mathrm{e}}_{2,3}^{b c 7}(-3)+\widetilde{\mathrm{e}}_{2,3}^{b c 8}+\widetilde{\mathrm{e}}_{2,3}^{b c 9}+\widetilde{\mathrm{e}}_{2,3}^{b c 10}+\sum_{\ell=1}^{7} \widetilde{\mathrm{e}}_{2,3}^{b c(7-\ell)} \ell \\
& =-171+93+202+400+114=638 \text {, } \\
& x(8)=\widetilde{\mathrm{e}}_{2,3}^{b c 8}(-3)+\widetilde{\mathrm{e}}_{2,3}^{b c 9}+\widetilde{\mathrm{e}}_{2,3}^{b c 10}+\widetilde{\mathrm{e}}_{2,3}^{b c 11}+\sum_{\ell=1}^{8} \widetilde{\mathrm{e}}_{2,3}^{b c(8-\ell)} \ell \\
& =-279+202+400+715+228=1266 .
\end{aligned}
$$

\section{Conflict of Interests}

The authors declare that there is no conflict of interests regarding the publication of this paper.

\section{Acknowledgment}

The first author was supported by the Grant 201/10/1032 of the Czech Grant Agency (Prague).

\section{References}

[1] J. Diblík and D. Ya. Khusainov, "Representation of solutions of linear discrete systems with constant coefficients and pure delay," Advances in Difference Equations, vol. 2006, Article ID 80825, 13 pages, 2006.

[2] J. Diblík and D. Ya. Khusainov, "Representation of solutions of discrete delayed system $x(k+1)=A x(k)+B x(k-m)+f(k)$ with commutative matrices," Journal of Mathematical Analysis and Applications, vol. 318, no. 1, pp. 63-76, 2006.

[3] J. Diblík and B. Morávková, "Discrete matrix delayed exponential for two delays and its property," Advances in Difference Equations, vol. 2013, article 139, 18 pages, 2013.

[4] D. Ya. Khusainov and G. V. Shuklin, "Linear autonomous timedelay system with permutation matrices solving," Studies of the University of Žilina. Mathematical Series, vol. 17, no. 1, pp. 101108, 2003.

[5] A. Boichuk, J. Diblík, D. Khusainov, and M. Růžičková, "Boundary value problems for delay differential systems," Advances in Difference Equations, vol. 2010, Article ID 593834, 20 pages, 2010.

[6] A. Boichuk, J. Diblík, D. Khusainov, and M. Růžičková, "Fredholm's boundary-value problems for differential systems with a single delay," Nonlinear Analysis: Theory, Methods \& Applications, vol. 72, no. 5, pp. 2251-2258, 2010.

[7] A. Boichuk, J. Diblík, D. Khusainov, and M. Růžičková, "Boundary-value problems for weakly nonlinear delay differential systems," Abstract and Applied Analysis, vol. 2011, Article ID 631412, 19 pages, 2011.

[8] J. Diblík, M. Fečkan, and M. Pospíšil, "Representation of a solution of the Cauchy problem for an oscillating system with two delays and permutable matrices," Ukrains'kyi Matěmatyčhnyi Žhurnal, vol. 65, pp. 58-69, 2013.

[9] J. Diblík, M. Fečkan, and M. Pospíšil, "Representation of a solution of the Cauchy problem for an oscillating system with multiple delays and pairwise permutable matrices," Abstract and Applied Analysis, vol. 2013, Article ID 931493, 10 pages, 2013.

[10] J. Diblík, D. Khusainov, O. Kukharenko, and Z. Svoboda, "Solution of the first boundary-value problem for a system of autonomous second-order linear partial differential equations of parabolic type with a single delay," Abstract and Applied Analysis, vol. 2012, Article ID 219040, 27 pages, 2012.

[11] J. Diblík, D. Ya. Khusainov, J. Lukáčová, and M. Růžičková, "Control of oscillating systems with a single delay," Advances in Difference Equations, vol. 2010, Article ID 108218, 15 pages, 2010.

[12] J. Diblík, D. Ya. Khusainov, and M. Růžičková, "Controllability of linear discrete systems with constant coefficients and pure delay," SIAM Journal on Control and Optimization, vol. 47, no. 3, pp. 1140-1149, 2008.

[13] D. Ya. Khusainov, J. Diblík, M. Růžičková, and J. Lukáčová, "Representation of a solution of the Cauchy problem for an 
oscillating system with pure delay," Nonlinear Oscillations, vol. 11, no. 2, pp. 276-285, 2008.

[14] D. Ya. Khusainov and G. V. Shuklin, "On relative controllability in systems with pure delay," International Applied Mechanics, vol. 41, no. 2, pp. 118-130, 2005.

[15] M. Medved' and M. Pospíšil, "Sufficient conditions for the asymptotic stability of nonlinear multidelay differential equations with linear parts defined by pairwise permutable matrices," Nonlinear Analysis: Theory, Methods \& Applications, vol. 75, no. 7, pp. 3348-3363, 2012.

[16] M. Medved', M. Pospíšil, and L. Škripková, "Stability and the nonexistence of blowing-up solutions of nonlinear delay systems with linear parts defined by permutable matrices," Nonlinear Analysis: Theory, Methods \& Applications, vol. 74, no. 12, pp. 3903-3911, 2011.

[17] M. Medved' and L. Škripková, "Sufficient conditions for the exponential stability of delay difference equations with linear parts defined by permutable matrices," Electronic Journal of Qualitative Theory of Differential Equations, no. 22, pp. 1-13, 2012.

[18] M. Pospíšil, "Representation and stability of solutions of systems of functional differential equations with multiple delays," Electronic Journal of Qualitative Theory of Differential Equations, no. 54, pp. 1-30, 2012. 


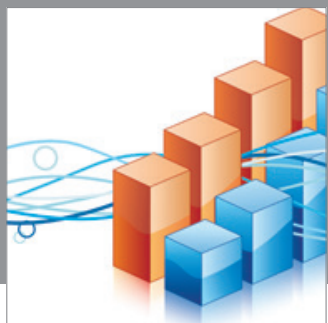

Advances in

Operations Research

mansans

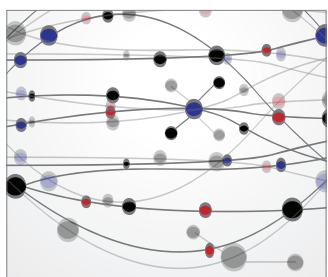

The Scientific World Journal
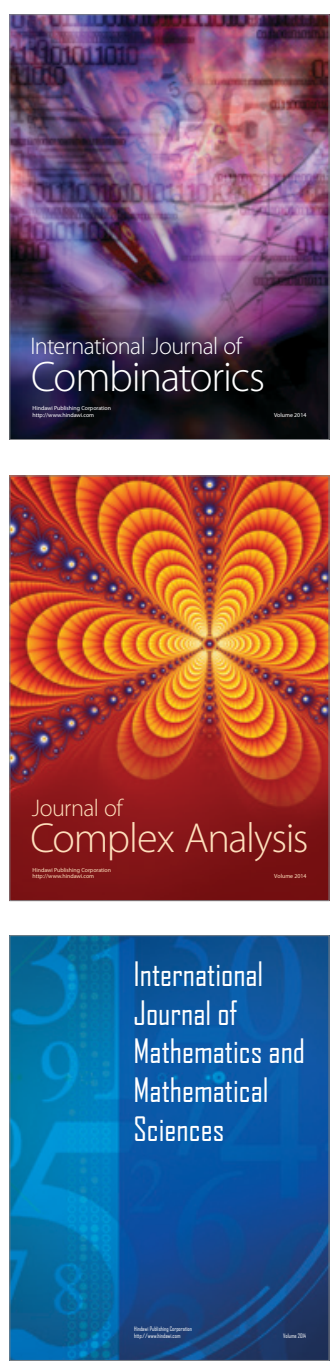
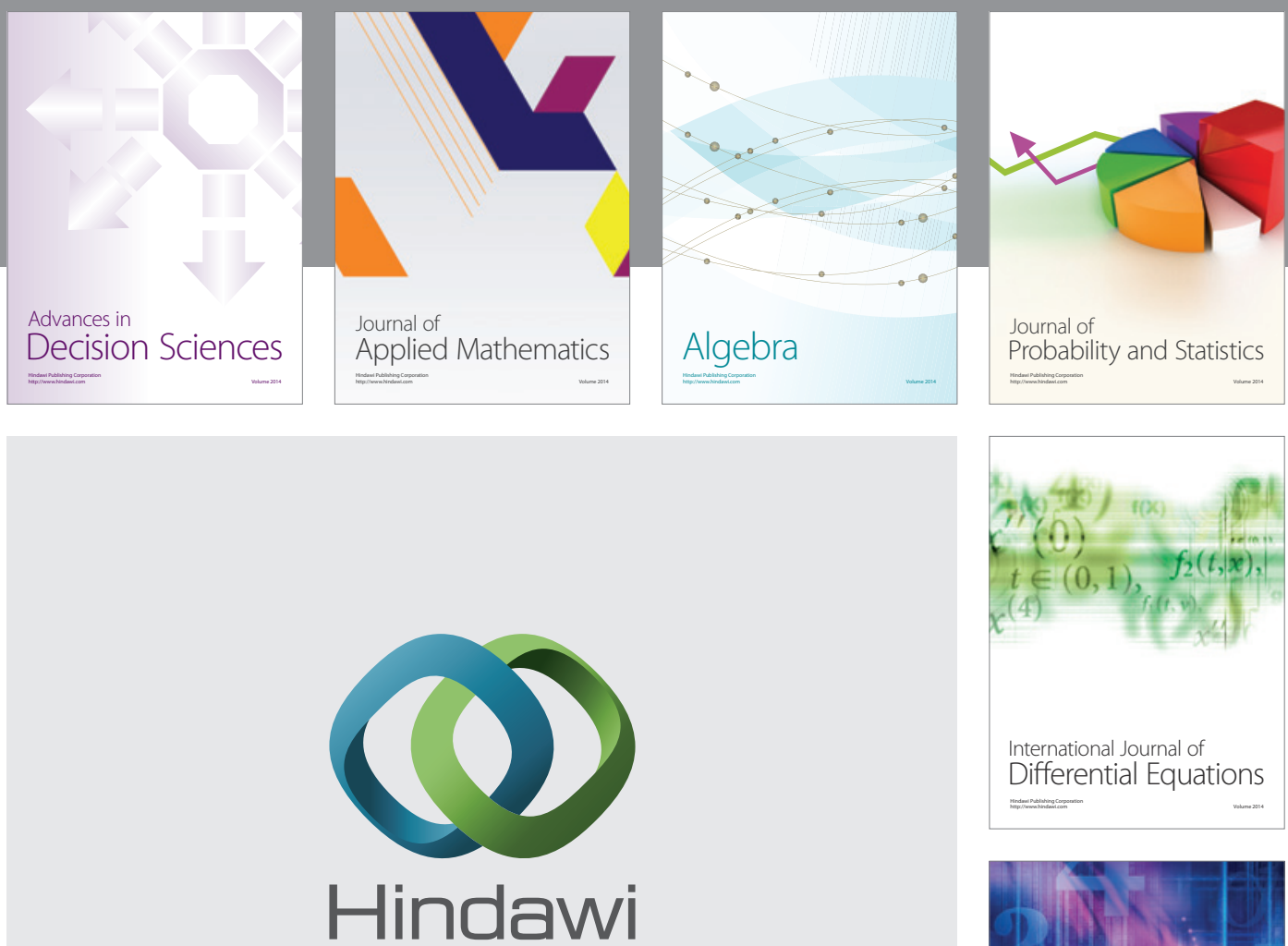

Submit your manuscripts at http://www.hindawi.com
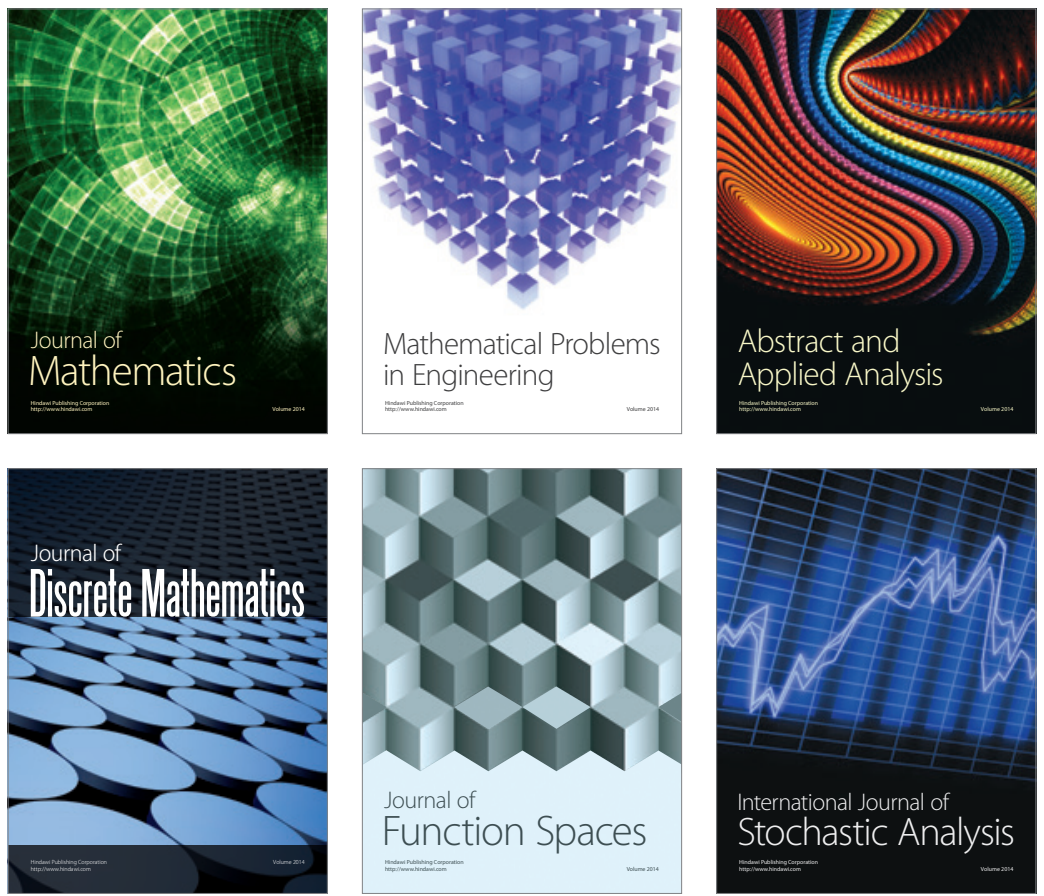

Journal of

Function Spaces

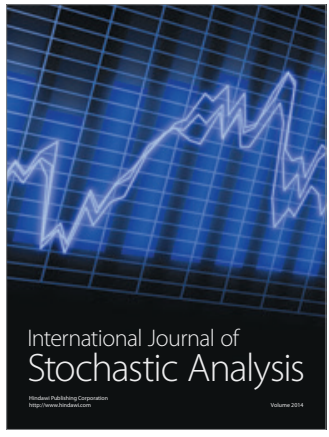

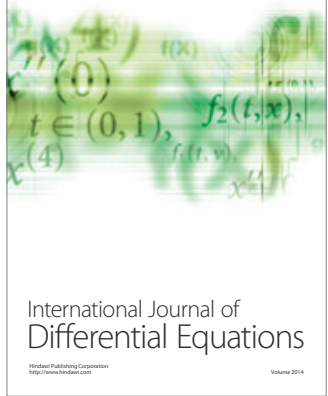
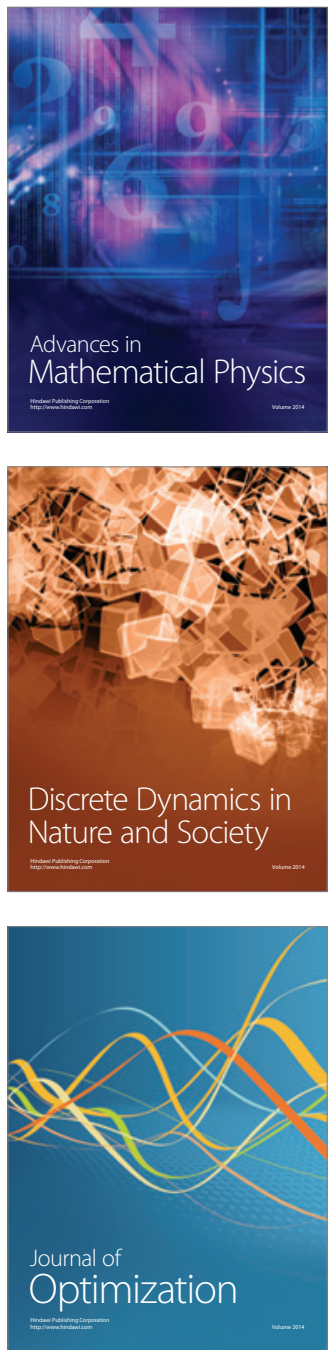